\title{
Greek High Phenolic Early Harvest Extra Virgin Olive Oil Reduces the Over-Excitation of Information-Flow Based on Dominant Coupling Mode Model in Patients with Mild Cognitive Impairment: An EEG Resting-State Validation Approach
}

\author{
Stavros I. Dimitriadis ${ }^{1-9^{*}}$, Christos Lyssoudis ${ }^{2}$, Anthoula C. Tsolaki ${ }^{1}$, Eftychia Lazarou ${ }^{2}$, \\ Mahi Kozori², Magda Tsolaki ${ }^{1,2}$ \\ ${ }^{1} 1$ st Department of Neurology, G.H. “AHEPA, " School of Medicine, Faculty of Health Sciences, Aristotle \\ University of Thessaloniki (AUTH), 54124 Thessaloniki, Greece \\ ${ }^{2}$ Greek Association of Alzheimer's Disease and Related Disorders, 54643 Thessaloniki, Makedonia, Greece \\ ${ }^{3}$ Integrative Neuroimaging Lab, 55133, Thessaloniki, Greece \\ ${ }^{4}$ Cardiff University Brain Research Imaging Centre (CUBRIC), School of Psychology, College of Biomedical \\ and Life Sciences, Cardiff University, CF24 4HQ, Cardiff, Wales, United Kingdom \\ ${ }^{5}$ Neuroinformatics Group, Cardiff University Brain Research Imaging Centre (CUBRIC), School of Psychology, \\ College of Biomedical and Life Sciences, CF24 4HQ, Cardiff, Wales, United Kingdom \\ ${ }^{6}$ Division of Psychological Medicine and Clinical Neurosciences, School of Medicine, College of Biomedical \\ and Life Sciences, Cardiff University, C CF24 4HQ, Cardiff, Wales, United Kingdom \\ ${ }^{7}$ School of Psychology, College of Biomedical and Life Sciences, CF24 4HQ, Cardiff, Wales, United Kingdom \\ ${ }^{8}$ Neuroscience and Mental Health Research Institute, School of Medicine, College of Biomedical and Life \\ Sciences, Cardiff University, CF24 4HQ, Cardiff, Wales, United Kingdom \\ ${ }^{9}$ MRC Centre for Neuropsychiatric Genetics and Genomics, School of Medicine, College of Biomedical and \\ Life Sciences, Cardiff University, CF24 4HQ, Cardiff, Wales, United Kingdom
}

\section{*Corresponding Email}

Dr. Stavros I. Dimitriadis

Research Fellow

Email:DimitriadisS@cardiff.ac.uk / stidimitriadis@gmail.com 


\begin{abstract}
:
Background: Extra virgin olive oil (EVOO) constitutes a natural compound with high protection over cognitive function that could positively alter brain dynamics and the mixture of within and between-frequency connectivity.
\end{abstract}

Objective: The balance of cross-frequency coupling (CFC) over within-frequency coupling (WFC) can build a nonlinearity index (NI) that encapsulates the over-excitation of information flow between brain areas and across experimental time. The present study investigated for the very first time how the Greek High Phenolic Early Harvest Extra Virgin Olive Oil (HP-EHEVOO) versus Moderate Phenolic (MP-EVOO) and Mediterranean Diet (MeDi) intervention in people with Mild Cognitive Impairment (MCI) could affect their spontaneous EEG dynamic connectivity.

Methods: Forty-three subjects (14 in MeDi, 16 in MP-EVOO, and 13 in HP-EH-EVOO) followed an EEG resting-state recording session (eyes-open and closed) before and after the treatment. Following our dominant coupling mode model (DoCM), we built a dynamic integrated dynamic functional connectivity graph (iDFCG) that tabulates the functional strength and the DoCM of every pair of brain areas.

Results: Signal spectrum within $1-13 \mathrm{~Hz}$ and theta/beta ratio have decreased in the HP-EHEVOO group in the eyes-open condition. The intervention improved the FI ${ }^{\text {DoCM }}$ across groups and conditions but was more prominent in the HP-EH-EVOO group ( $p<0.001)$. Finally, we revealed a significant higher post-intervention reduction of NI $\left(\Delta \mathrm{NI}^{\text {Total and } \alpha}\right)$ for the HP-EHEVOO compared to the MP-EVOO and MeDi groups ( $<<0.0001)$.

Conclusions: Long-term intervention with HP-EH-EVOO reduced the over-excitation of information flow in spontaneous brain activity and altered the signal spectrum of EEG rhythms. 


\section{Highlights:}

- Non-pharmaceutical intervention based on HP-EH-EVOO in MCI reduces the overexcitation of information flow.

- Non-pharmaceutical intervention based on HP-EH-EVOO in MCI increases the human brain flexibility.

- Alpha frequency modulates the reconfiguration of dominant coupling modes in EEG resting-state due to the intervention.

\section{Keywords:}

Alzheimer's disease

Mild cognitive impairment

EEG

Multiplexity

Chronnectomics

Cross-frequency coupling

extra virgin olive oil, Mediterranean Diet

non-pharmaceutical interventions

\section{Introduction}


A multifactorial syndrome that depends on psychological, neurobiological, and social factors contributing to a high risk for developing dementia characterizes mild cognitive impairment (MCI) [1] . Clinical judgment of MCI demands multiple sessions and multiple domain tests to detect a cognitive decline and impairment across single or various cognitive functions [2] . Distinctions over multiple (amnestic or non-amnestic) and single-domain MCI are more than significant, bearing the fact that amnestic-MCI single-domain, amnestic-MCI multiple-domain, and mild Alzheimer's disease (AD) could represent a continuum process that leads to deviations from normal aging [3].

Besides the heterogeneity of pathophysiological mechanisms underlying MCI subjects and their diagnostic misidentification, there are no pharmaceutical therapeutic options available and approved by FDA or EMA. The pharmaceutical interventions include acetylcholinesteraseinhibitors and memantine, which are approved only for $\mathrm{AD}$ and not for $\mathrm{MCI}$, and other medications that are not yet approved, such as ginkgo biloba, anti-inflammatory drugs, statins, platelet aggregation inhibitors (trifusal), piracetam, and others [4] . However, clinical trials based on the substances mentioned above targeting neurofibrillary tangle formation and amyloid-beta accumulation failed to show a high efficient performance measure for $A D$ patients with primary and secondary cognitive parameters [5] .

Recent evidence from non-pharmaceutical interventions in MCI and dementia subjects opens new avenues over alternative treatments [6]. Recent studies reported promising evidence of alternative non-pharmaceutical treatments for MCI based on natural substances (e.g., Extra Virgin Olive Oil (EVOO) [7], saffron [8]) and dietary interventions (e.g., the Mediterranean diet (MeDi)) [9];. The MeDi is not a prescribed diet by a clinician but a general eating habituation from people located geographically around the Mediterranean sea. High consumption of olive oil, vegetables, legumes, fruits, unprocessed cereals, moderate to high consumption of fish, low consumption of meat and meat products, moderate wine consumption, 
and low to moderate consumption of dairy products characterize the traditional Mediterranean diet. Scarmeas et al. reported that the adaptation of the MeDi diet for a long time improved various cognitive functions and decreased the risk of developing MCI and progress from MCI to $\mathrm{AD}[10]$. Moreover, healthy dietary habituations are strongly correlated with positive cognitive outcomes [11].

Researchers evaluated positive evidence of an EVOO-enriched diet against AD pathology in both animals and humans. Researchers explored if the consumption of an EVOO-enriched diet from TgSwDI mice could reduce the accumulation of amyloid- and tau-related brain levels and related alterations of cognitive functions. They reported that a long-term (6 months) consumption of an EVOO-containing diet provided a protective mechanism against AD pathology [12]. A clinical study investigated the potentially positive role of EVOO-rich MeDi in 285 subjects with high vascular risk. The authors reported improved cognitive functioning compared to a control diet. Additionally, participants who followed EVOO-rich MeDi had fewer MCI subjects at follow-up than the controls, while participants who followed a MeDi + Nuts did not differ from the controls in terms of the number of MCI subjects [13] after seven years of follow up.

The main ingredients of EVOO are glycerides of fatty acids (98\%), mainly of monounsaturated fatty acids (MUFA), and especially oleic acid. The remaining $2 \%$ include various "minor compounds" like phenolic alcohols, phenolic acids, lignans, flavones, and, importantly, secoiridoids and their derivatives $[14,15]$. Natural phenols can modulate cell redox state [16] via a direct activity over enzymes, proteins, receptors, and various signaling pathways [17,18]. Another positive impact of natural phenols is their interference with biochemical homeostasis [19,20], which further impacts epigenetic alterations of the chromatin $[21,22]$. 
There is strong evidence that MeDi prevents neurodegeneration due to the consumption of vegetables, legumes, and olive oil rich in polyphenols [10,23-26]. Phytochemicals, which are chemical compounds produced by plants like flavonols, support the cell's different protective biological activity. The repertoire of this activity could be anti-oxidant, anti-allergic, antiinflammatory, anti-viral, anti-carcinogenic, and anti-proliferative [27-29]. MeDi includes, in addition to fruits and vegetable consumption, consumption of EVOO in amounts ranging from 25 to 50 g/day $[12,30,31]$. Furthermore, studies reported that polyphenols act as an antiaggregation agent against the disposition of amyloid- $\beta_{1-42}$ peptide plaques supporting an antitoxic environment [32], while oleocanthal can alter the oligomerization state of oligomers of amyloid- $\beta_{1-42}$ peptide, which are toxic [33]. Thus, both substances could have a significant impact on the modifying treatment of AD.

A study evaluated the link between cognitive performance and dietary habits [34], while it associated oxidative stress with neurodegenerative disorders and cognitive decline [35]. A simple hypothesis from the evidence above of the beneficial properties of antioxidant-rich foods is that their consumption could reduce oxidative stress, which could further protect from neurodegenerative diseases. MeDi is full of antioxidant-rich foods with the primary origin of plants and associated with health status [36].

Normally, cells can counteract the oxidative insults by regulating their homeostasis in neurodegenerative conditions related to aging. During aging, the cells' capacity to maintain a redox balance decreases, which further influences mitochondrial dysfunction, neuronal injury, and the accumulation of radicals. We know that oxidative stress increases with aging [37], and it is a significant age factor that makes neuronal systems more vulnerable to various neurodegenerative diseases [38]. Oxidative stress causes mitochondrial injury, which further causes loss of oxidative metabolism and overproduction of free radicals. Oxidative stress contributes to many human diseases such as AD [39] and Parkinson's disease [40]. 
Our MICOIL Pilot Study was the first-ever reported longitudinal double-blind study administered Greek HP-EH-EVOO to people with MCI for one year. The purpose of MICOIL was to evaluate the effect of a Greek HP-EH-EVOO+MeDi (Group 1) compared to a Greek MP-EVOO+MeDi (Group 2) and MeDi only (Group 3) on the multi cognitive status of patients with MCI, who are community-dwelling, Greek-speaking elderly, with age ranging from 60 to 80 [7]. The Greek HP-EH-EVOO received (50mL/day) together with MeDi instructions, the GreekMP-EVOO received (50mL/ day), and MeDi instructions and MeDI group received only the MeDi instructions. The evaluated hypothesis was that long-term intervention with either HP-EH-EVOO or MP-EVOO could significantly improve cognitive functions compared to MeDi, independently of APOE $\varepsilon 4$ carrier.

In the present study, we analyzed electroencephalographic (EEG) resting-state recordings (both eyes-open and eyes-closed conditions) from a subset of participants from the three groups compared to the previous study focusing only on neuropsychological estimates [7]. We recorded brain activity with EEG before and after the intervention with the three groups. We adopted a dynamic functional connectivity analytic pathway to incorporate our dominant coupling mode model (DoCM). Our DoCM incorporated both within frequency (intrafrequency) and between frequencies (cross-frequency) functional interactions under the same model. The primary outcome of DoCM is the construction of an integrated dynamic functional connectivity graph (iDFCG) that tabulates both the functional strength and the DoCM of every pair of brain areas across experimental time [41]. When two brain areas communicate under the same frequency content (intra-frequency), this exchange of information is called linear. However, cross-frequency interactions are non-linear information pathways and are highly active when two functionally distinct brain areas want to interact and exchange information [42]. The ratio of cross-frequency over intra-frequency interactions can build a nonlinearity index (NI) of information change in our brain. In a resting-state EEG study, we showed in a 
group of adolescents with schizophrenia spectrum disorders (SSDs) that NI is significantly higher than an age-matched healthy group, and the alpha frequency drives the reconfiguration of dominant coupling modes [43]. Here, we will adopt the same methodology assuming that the three groups will reduce their individual NI after the treatment, and also group1 and two administered with Greek HP-EH-EVOO or Greek MP-EVOO will show a more substantial reduction of their NI compared to the MeDi group 3. We hypothesized that a change of NI suggests reducing cross-frequency coupling (CFC) non-linear interactions, and the alpha frequency band will drive these changes, a frequency of arousal at resting-state conditions [44]. Eyes-open and eyes-closed are not two equivalent baseline ones, and we will analyze them as two different conditions. The eyes-closed condition is an arousal baseline, while the eyes-open condition is an activation baseline.

\section{Material and Methods}

\subsection{MICOIL Study Design}

From 1 December 2016 to 30 August 2018, participants were recruited from the Memory \& Dementia clinic of the 3rd and 1st Neurology Departments of Aristotle University of Thessaloniki, Greece, and the two Day Centers of the Greek Association of Alzheimer's Disease and Related Disorders (GADRDA). The MICOIL study followed the ethical principles as described in the Declaration of Helsinki for human experimentation. GADRDA's scientific \& ethics committee (25/ 240 2016) (Clinical Trials Registration Number: NCT03 241 362996) ${ }^{1}$ approved the study. All participants fulfilled the Petersen criteria for MCI [45]. Information about inclusion and exclusion criteria, study design, APOE genotype, randomization and allocation procedure of participants' selection, withdrawals, and description of every protocol

\footnotetext{
${ }^{1}$ https://clinicaltrials.gov/ct2/show/NCT03362996
} 
followed for each group. We forward an interested reader to the first report of the MICOIL study [7].

We adopted the following protocol to instruct our participants on the MeDi diet and monitor their compliance. We first gave them a form with the food they can eat, including a wide variety of the Mediterranean diet. Each time we supported oil to the subjects, we asked specific questions to make sure they followed the Mediterranean diet. From time to time, we also asked the same questions to the control group.

There were three questions.

1. Did you follow the Mediterranean diet instructions we gave you?

2. Is your daily diet determined according to the list of foods allowed in the Mediterranean diet?

3. With your hand on your heart, what $\%$ did you follow this diet? $50 \%, 80 \%$ or $100 \%$

The Average compliance was higher than $80 \%$ across the groups without any statistical group difference $(\mathrm{p}<0.05)$

\subsection{Participants}

Table 1 tabulates the demographics of the subjects that participated in EEG data collections. Following a Kruskal-Wallis non-parametric test, we did not detect any effect on age, gender ratio, and education level. 
Table 1. Mean and standard deviation (SD) of demographics of all participants, and the results of the non-parametric Kruskal-Wallis statistical test

\begin{tabular}{|c|c|c|c|c|c|}
\hline & $\begin{array}{l}\text { MeDi } \\
(n=14) \\
\text { mean } \pm \text { std }\end{array}$ & $\begin{array}{l}\text { MP-EVOO } \\
(n=16) \\
\text { mean } \pm \text { std }\end{array}$ & $\begin{array}{l}\text { HP-EH- } \\
\text { EVOO } \\
(n=13) \\
\text { mean } \pm \text { std }\end{array}$ & $\begin{array}{l}\text { All } \\
(n=43) \\
\text { mean } \pm \text { std }\end{array}$ & $\begin{array}{l}\text { Kruskal- } \\
\text { Wallis test } \\
H(d f)=x^{2}, p\end{array}$ \\
\hline Age (Years) & $70.5 \pm 5.9$ & $71.2 \pm 7.9$ & $69.5 \pm 7.6$ & $70.5 \pm 7.1$ & $\begin{array}{l}H(2)=0.47 \\
\mathbf{p}=\mathbf{0 . 7 8}\end{array}$ \\
\hline $\begin{array}{l}\text { Gender } \\
(\mathrm{F}: M)\end{array}$ & $12: 2$ & $11: 5$ & $7: 6$ & $30: 13$ & $\begin{array}{l}\mathrm{H}(2)=3.18 \\
\mathbf{p}=\mathbf{0 . 2 0}\end{array}$ \\
\hline $\begin{array}{l}\text { Education } \\
\text { (Years) }\end{array}$ & $10.9 \pm 4.7$ & $9.1 \pm 3.6$ & $11.4 \pm 3.0$ & $11.4 \pm 3.0$ & $\begin{array}{l}\mathrm{H}(2)=3.29 \\
\mathrm{p}=\mathbf{0 . 1 9}\end{array}$ \\
\hline
\end{tabular}

[Table 1 around here]

\subsection{Neuropsychological Assessment}

The neuropsychological battery included: the Greek version of Mini-Mental State Examination (MMSE) to assess the general cognitive function, the Rivermead Behavioral Memory Test-Story including the Immediate and Delayed recall for episodic memory, the Rey Osterrieth Complex Figure Test copy, and delayed recall which measures long-term visuospatial memory and executive functions, the Trail Making Test parts A \& B, to examine visuospatial ability, attention and executive functions, the Alzheimer Disease Assessment Scale-Cognition (ADAS-Cog) to assess the severity of cognitive dysfunction, the Wechsler 
Memory Scales Digit Span Forward and Backward to assess attention and working memory, the Letter, and Category Fluency Test for assessing phonemic and semantic fluency and the Clock-drawing Test which measures visuospatial orientation, understanding of verbal instructions, abstract thinking, planning, concentration, executive and visuospatial skills. In addition, depressive symptoms were assessed by the Geriatric Depression Scale using a cut-off score of $<6$ at baseline. Finally, we also used the Neuropsychiatric Inventory to assess other neuropsychiatric symptoms since it is a critical component for evaluating the MCI subjects because their distress can cause or exacerbate cognitive problems. For further details regarding the neuropsychological assessments, see the first paper focusing on neuropsychological estimates [7].

\subsection{EEG Recordings}

We performed EEG experiments in cognitively intact elderly subjects and subjects suspected of neurodegenerative disorder and cognitive deficits. To reduce the time of the EEG montage, we should use a minimum of 19 standard exploring electrodes placed according to the primary international 10-20 system (i.e., Fp1, Fp2, F7, F3, Fz, F4, F8, T3, C3, Cz, C4, T4, T5, P3, Pz, P4, T6, O1, and O2). For all EEG recordings, there was the inclusion of two ears (A1 and A2). Therefore, the resistance of the EEG electrodes should be lower than $5 \mathrm{KOhm}$. EEG experiment included with the resting state EEG conditions:

1) Resting-state eyes closed EEG recording for 5 minutes (ideally, most of these 5 minutes of EEG recordings should be characterized by a subject relaxed, not showing voluntary or involuntary movements).

2) Resting-state eyes open EEG recording for 5 minutes.

We set the sampling frequency to fs $=500 \mathrm{~Hz}$. 
EEG time series were re-referenced to the average reference electrode [46] before preprocessing steps. The local ethical committee has approved the experiments of Alzheimer Hellas (no. 25/21-6-2016).

\subsection{Artifact reduction with independent component analysis (ICA) and Wavelet}

\section{Decomposition}

An important pre-processing step before estimating a dynamic functional connectivity graph (dFCG) is denoising EEG recordings. Therefore, we adopted an already established algorithmic artifact reduction method that combines independent component analysis (ICA) and Wavelet Decomposition. For further details, see our recent study, which describes the adopted denoising approach [43]. Briefly, most studies adopted ICA that produces $\mathrm{N}$ independent components (ICs) where $\mathrm{N}$ denotes the number of EEG sensors. Afterward, we rejected ICs as artifactual components based on the time course of ICs, the topology of the related weights per sensor, and estimating measures like entropy, kurtosis, and skewness to support our decision further. The main drawback of this approach, especially for resting-state, is that we have to zero one IC as a whole even if we detected any artifact in specific epochs covering a small percentage of the total experimental time. For that reason, we decided in our previous study to decompose the time course of artifactual IC in epochs of $1 \mathrm{sec}$ using wavelet decomposition. This approach gives us an advantage over the traditional approach, avoiding rejecting an IC that encapsulates critical actual brain activity mixed with many artifactual epochs [43].

\subsection{Signal Power Analysis}

We estimated power spectral density (PSD) using the pwelch MATLAB function independently for every EEG sensor for each subject before and after the intervention and in 
both conditions. Then, we averaged PSD estimates across EEG sensors to characterize every subject's PSD profile for every condition and in both pre and post-intervention periods. We analyzed the PSD up to $45 \mathrm{~Hz}$. We also estimated the frontal theta/beta ratio as a marker of attentional control [47] [48].

\subsection{The integrated dynamic functional connectivity graph (IDFCG) based on iPLV}

This section will briefly describe our dominant intrinsic coupling model (DoCM) demonstrated in the whole repertoire of functional neuroimaging modalities $[41,43,49-58]$. Most studies investigated functional connectivity independently for every frequency band while focusing mainly on within-frequency (intra-frequency) interactions. Last year, an increased amount of research studies also explored functional connectivity between brain areas oscillating on a different frequency, the so-called cross-frequency coupling (CFC) or interfrequency interactions. DoCM model incorporates all possible functional connectivity modes under the same framework. The main aim of the DoCM model is to detect the dominant coupling mode between every pair of EEG sensors and across temporal segments. Here, we adopted intra-frequency phase-to-phase and inter-frequency phase-to-amplitude potential coupling modes (CFC). We estimated dynamic functional connectivity graph (dFCG) within and between the seven studying frequency bands $\left\{\delta, \theta, \alpha_{1}, \alpha_{2}, \beta_{1}, \beta_{2}, \gamma\right\}$ defined, respectively, within the ranges $\{0.5-4 \mathrm{~Hz} ; 4-8 \mathrm{~Hz} ; 8-10 \mathrm{~Hz} ; 10-13 \mathrm{~Hz} ; 13-20 \mathrm{~Hz} ; 20-30 \mathrm{~Hz} ; 30-48 \mathrm{~Hz}\}$. For this computation, we employed the EEG activity from the 19 EEG sensors in both restingstate conditions. EEG recordings were bandpass filtered using a $3^{\text {rd }}$ order zero-phase Butterworth filter employing the filtfilt MATLAB function. The width of the temporal window was set equal to $500 \mathrm{~ms}$ (or 250 samples) and moved forward across experimental time with a step equal to $100 \mathrm{~ms}$ (50 samples), which can encapsulate both slow and fast oscillations 
$[41,43,49-58]$. We finally analyzed 75 secs across subjects and conditions defined as the maximum consistent experimental time across the participants.

For every pair of EEG sensors and every temporal segment, we estimated the seven intrafrequency phase-to-phase possible interactions, one for every frequency band, and twenty-one inter-frequency phase-to-amplitude interactions between every possible pair of the seven frequency bands. Here, we adopted iPLV as the proper connectivity estimator for both intra and inter-frequency coupling modes as in our previous studies. In total, we estimated twentyeight possible coupling modes leading to twenty-eight dFCG per subject and condition. A $\mathrm{dFCG}$ is a 3D matrix of dimensions [temporal segments $\mathrm{x}$ sensors $\mathrm{x}$ sensors]. Then, we followed a surrogate analysis with the main scope to detect the dominant coupling mode per pair of EEG sensors and for each temporal segment (for further details, see [43]). This approach leads to the integrated dFCG (DIFCG) that keeps both the strength and the dominant coupling mode per pair of EEG sensors and experimental time. The IDFCG is a pair of 3D matrices of size [temporal segments $\mathrm{x}$ sensors $\mathrm{x}$ sensors] where one keeps the strength and the other the dominant coupling mode. The functional strength ranges $[0,1]$ based on the adopted connectivity estimator iPLV while we encoded the dominant coupling mode with an integer from 1 up to 28 , e.g., 1 for $\delta, 2$ for $\theta, 3$ for $\alpha_{1}, \ldots, 26$ for $\beta_{1-} \beta_{2}, 27$ for $\beta_{1-\gamma}$ and 28 for $\beta_{2}-\gamma$.

Fig. 1 is created in analogy to previous studies to exemplify the DoCM model's concept is not familiar to readers [43] [58]. Fig.1A illustrates how DoCM works for the first two temporal segments of eyes-closed condition from the first subject of group A between Fp1 and Fp2 EEG sensors. Following the critical step of the surrogate analysis, we revealed $\alpha_{1}-\beta_{2}$ cross-frequency phase-to-amplitude coupling (PAC) as the dominant coupling mode (DoCM) among 28 possible coupling modes for the first two temporal segments for this particular EEG pair of sensors. On the right side of frequency-dependent pairs of time series, we demonstrated the functional strength measured with iPLV OF every possible coupling mode as a matrix. The 
main diagonal of this matrix tabulates the intra-frequency phase-to-phase coupling modes, while the off-diagonal stores the cross-frequency phase-to-amplitude coupling modes. Fig.1B illustrates the temporal evolution of DoCM for the Fp1-Fp2 EEG pair. The color encodes the functional strength of the coupling, while the y-axis refers to the detected DoCM. We showed the probability distribution (PD) of DoCM across experimental time on the right side of this semantic time series. From this representation, $\alpha_{1}-\alpha_{1}$ intra-frequency phase-to-phase coupling was the most representative across DoCM. The flexibility index (FI) and PD are estimated from the semantic time series, as shown in Fig. 1B.

A

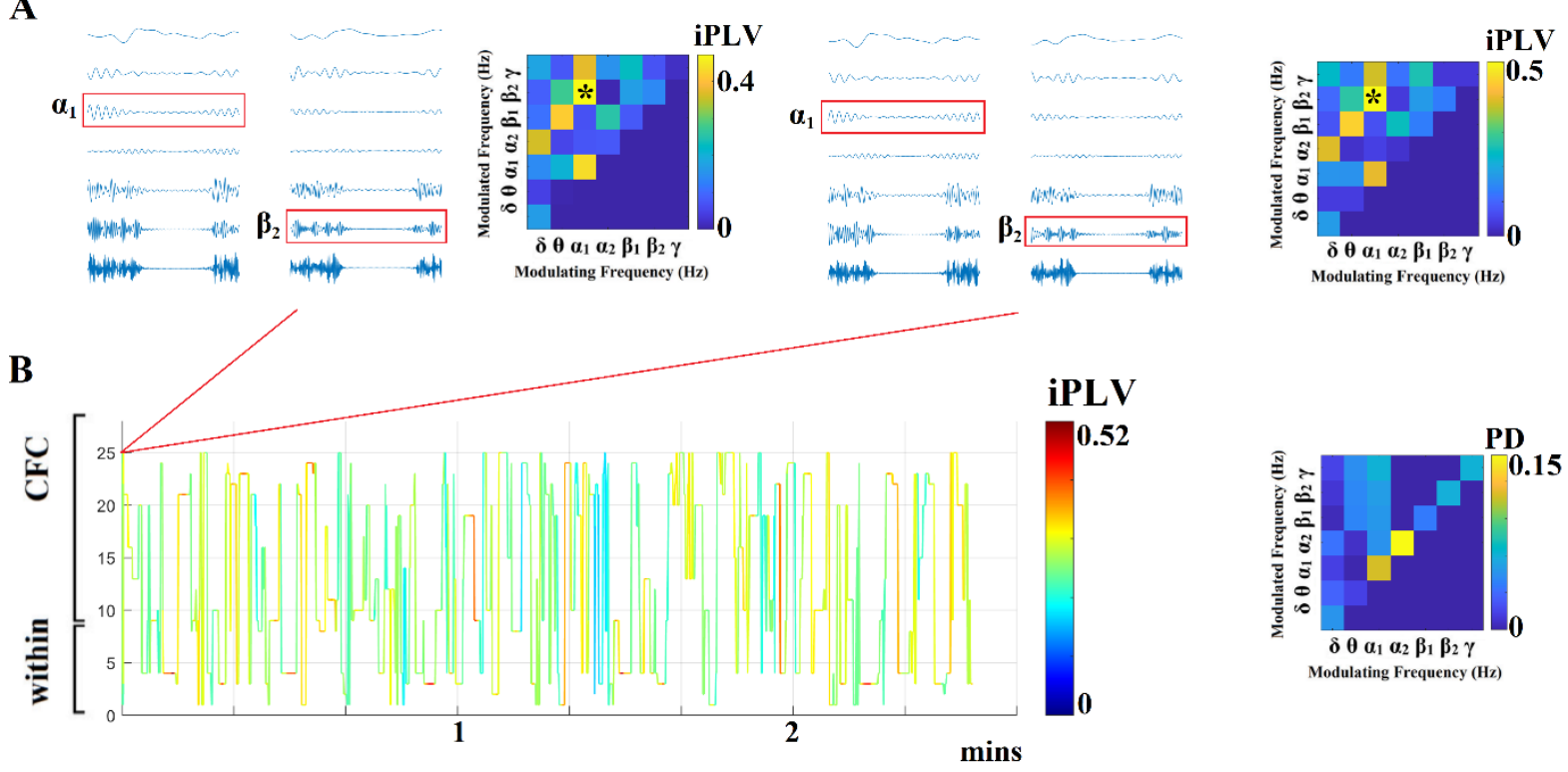

Figure 1. Detecting Dominant Intrinsic Coupling Modes (DoCM) based on iPLV.

(A) A schematic illustration of the adapted DoCM model showing the detection of dominant coupling mode between EEG sensors Fp1 and Fp2 for the first two consecutive temporal segments (ts1, ts2). Surrogate analysis revealed the DoCM for both temporal segments. During 
both ts 1 and ts2, the DoCM reflected significant phase-to-amplitude coupling between $\alpha_{1}$ phase and $\beta_{2}$ amplitude (indicated by red rectangles). Both colored matrices tabulate the functional strength quantified with iPLV of both intra-frequency coupling modes (main diagonal) and inter-frequency coupling modes (off-diagonal).

(B) Burst of DoCM between the Fp1 and Fp2 sensors. The colored time series is the streaming of information flow between two brain areas where the main elements of this neural communication are the DoCM. We could interpret this streaming of DoCM as a temporal messaging of brain areas at the macroscale level. The y-axis in B. encodes the DoCM dividing the axis into intra and inter-frequency coupling modes. The matrix on the right tabulates the probability distribution (PD) of DoCM for the semantic time series on the left. $\alpha_{1}-\alpha_{1}$ phase-tophase intra-frequency coupling was the most prominent among 28 potential coupling modes.

We estimated the flexibility index (FI) over a semantic time series shown in B. by quantifying the number of times a DoCM changed between consecutive temporal segments divided by the number of temporal segments -1 . We interpreted high values of FI, which ranges within $[0,1]$, as functionally more flexible communication between brain areas.

[Figure 1 around here]

\subsection{Semantic features derived from the evolution of DoCM}

We describe in this section the extraction of semantic features from the $2^{\text {nd }} 3 \mathrm{D}$ tensor that preserves the DoCM across EEG sensor space and experimental time. 


\subsubsection{Flexibility index (FI)}

We estimated the Flexibility index (FI), a measure that quantifies the transition rate of DoCM between every pair of EEG sensors $[43,53,56,58]$. We employed the $2^{\text {nd }} 3 \mathrm{D}$ tensor of the DIFCG that tabulates the semantic information of DoCM across the brain and experimental time to estimate FI. Fig.1B shows an example of such a tensor and what information it tabulates.

We call this metric hereafter $\mathrm{FI}^{\mathrm{DoCM}}$, and the following equation describes it:

$$
F I^{\text {DoCM }}=\frac{\text { no of transitions }}{\text { temporal segments }-1}
$$

We estimated the $\triangle F I^{D o C M}$ between pre and post-intervention $F I^{D o C M}$

$$
\Delta F I^{\text {DoCM }}=\frac{F I_{\text {post }}^{\text {DoCM }}-F I_{\text {pre }}^{\text {DoCM }}}{F I_{\text {pre }}^{\text {DoCM }}}
$$

We counted a transition only when a coupling exists between two consecutive temporal segments. The denominator (temporal segments -1 ) equals the pairs of temporal segments where a functional coupling mode exists. It is essential to underline that the FI measure counts only the change of the DoCM across time and not the preferred transition between specific pairs of frequency coupling modes, while $\mathrm{FI}^{\mathrm{DoCM}}$ gets higher values for higher "transitions" of DoCM between neighboring in-time temporal segments. We illustrated an example of the estimation of $\mathrm{FI}^{\mathrm{DoCM}}$ for the Fp1-Fp2 EEG pair in Fig. 1B. The outcome of FI estimation for every pair of EEG sensors is a matrix of size $19 \times 19$. Then, we estimated the nodal FI as the mean of every row of this matrix. Finally, we estimated the global FI by averaging the 19 nodal FI. 


\subsubsection{Spatiotemporal distribution of DoCM-Comodulograms}

Based on the $2^{\text {nd }} 3$ D DIFCG that keeps the semantic information of the preferred dominant coupling mode, we can tabulate in frequencies $\times$ frequencies matrix the probability distribution (PD) of observing each of the DoCM frequencies across 7 (intra-frequency) +21 (crossfrequency coupling) $=28$ possible coupling modes. We estimated PD across sensor space between the EEG sensor pairs and also across temporal segments.

The spatiotemporal PD tabulated in a matrix is called hereafter comodulogram, and an example is demonstrated in Fig. 1B [49],[50],[53]-[55,56].

\subsubsection{Nonlinearity index (NI) based on DoCM}

When two brain areas communicate within the same frequency (intra-frequency), then this communication is linear. However, when two brain areas communicate via cross-frequency coupling, this pathway is non-linear and plays a pivotal role in inter-areal communication $[43,59,60]$. The distinction between within-frequency coupling (linear coupling) and crossfrequency coupling (non-linear coupling) has been validated via biophysical modeling [59],[60]. Non-linear information pathways are highly active when two brain areas want to exchange information [42].

As was mentioned in section 2.7.2, we estimated the PD of DoCM across EEG sensor space and at every temporal segment. Therefore, we can divide the PD into two sections, the intrafrequency interactions ( 7 in total) stored in the main diagonal in the comodulogram (Fig.1B) and the cross-frequency interactions tabulated in the off-diagonal (21 in total).

The sum of these 21 cross-frequency PD values versus the seven intra-frequency values defines our nonlinearity index (NI) [43] described in eq. 2. In our study, as in a previous one [43], we assumed that $\alpha$ frequency should be the primary driving frequency of any 
improvement (reduction) of NI after the intervention at the resting-state conditions. To quantify the driving role of $\alpha$ frequency, we estimated NI as the ratio of the sum of PDs between $\alpha 1$ and $\left\{\alpha_{2}, \beta_{1}, \beta_{2}, \gamma\right\}$ (4 PDs related to 4 cross-frequency coupling pairs) and between $\alpha_{2}$ and $\left\{\beta_{1}, \beta_{2}, \gamma\right\}$ (3 PDs related to 3 cross-frequency coupling pairs) with the sum of PDs related to $\alpha_{1}$ and $\alpha_{2}$ within frequencies interactions (2 PDs related to 2 intra-frequency couplings) (eq. 3). The higher the NI, the higher is the contribution of CFC to the DoCM, and so the higher is the nonlinear communication between brain areas. The outcome of this process is a time series of size equal to the number of temporal segments, which is hereafter will be called dynamic NI (dNI). Two dNI were estimated per subject and condition, one incorporating the whole repertoire of coupling modes and one targeting the $\alpha$ frequency as a modulating frequency. The eq. 3 and 4 report the estimation of $\mathrm{NI}^{\text {Total }}$ and $\mathrm{NI}^{\alpha}$ for one temporal segment.

$$
\begin{gathered}
N I^{T \text { TOTAL}}=\frac{\sum_{n=1}^{\# \text { of CFC }} P D^{C F C}}{\sum_{k=1}^{\# \text { of within }} P D^{\text {within }}}(3) \\
N I^{\alpha}=\frac{P D^{a 1-a 2}+P D^{a 1-\beta 1}+P D^{a 1-\beta 2}+P D^{a 1-\gamma}+P D^{a 2-\beta 1}+P D^{a 2-\beta 2}+P D^{a 2-\gamma}}{P D^{a 1}+P D^{a 2}}
\end{gathered}
$$

To quantify the potential difference of dNI between the pre and post-condition, we estimated the delta difference $\Delta$ of the median values of dNI.

$$
\Delta N I^{\text {Total or } \delta, \theta \alpha, \beta}=\frac{\text { median }\left(d N I_{\text {pot }}^{\text {Total or } \delta, \theta \alpha, \beta}\right)-\text { median }\left(d N I_{\text {pre }}^{\text {Total or } \delta, \theta \alpha, \beta}\right)}{\text { median }\left(d N I_{\text {pre }}^{\text {Total or } \delta, \theta \alpha, \beta}\right)}(5)
$$

\subsection{Statistical Analysis}

We estimated Cohen's d effect size for PSD as a mean across frequency bin between pre and post-intervention period per group and in both conditions. We applied a Wilcoxon Signed Ranks Test to the theta/beta ratio between pre and post-intervention periods per group. We also 
performed statistical analysis between pairs of groups for detecting group differences in terms of $\Delta$ NI. We also applied statistical analysis over PD of DoCM independently per group between pre and post-intervention conditions to uncover the reorganization of DoCM due to intervention. For statistical comparisons over $\triangle \mathrm{NI}$ and PD of DoCM, we adopted the one-way ANOVA with $\mathrm{p}<0.05$ corrected for multiple comparisons.

We estimated the delta $(\Delta)$ difference of each neuropsychological assessment for FI and NI with the following formula (post - pre)/pre. $\Delta \mathrm{NI}^{\text {Total or } \alpha}$ from the eyes-open condition were used as the response variable and neuropsychological assessments as predictors following multi-linear regression analysis. We excluded clock drawing, clock copy, digit span forward, and digit span backward from the analysis due to many stable findings in the pre and postintervention period $(\Delta=0)$. We followed the above analysis in both resting-state conditions even though the eyes-open condition is more appropriate as it matches the condition of the neuropsychological session.

\subsection{Power Analysis}

We estimated effect size and actual power analysis a posteriori on the estimated measurements of global $\Delta \mathrm{FI}^{\mathrm{Total}}$ and $\Delta \mathrm{NI}^{\mathrm{Total} \text { or } \alpha}$. As a statistical test, we employed a one-way ANOVA omnibus with fixed effects. First, we set the power ( $1-b$ error probability) to 0.95 and the $\alpha$ error probability to 0.05 . Then, we repeated the analysis independently for the four conditions ( $\{$ eyes-open - eyes-closed $\left.\} \times\left\{\mathrm{NI}^{\mathrm{Total}}, \Delta \mathrm{NI}^{\alpha}\right\}\right)$.

\subsection{Software}

We analyzed EEG recordings in the MATLAB environment (v2019b) using the signal processing toolbox while we adopted Fast ICA from the fieldtrip toolbox. Dynamic functional 
connectivity analysis based on in-house software provided on our GitHub website: https://github.com/stdimitr/time_varying_PAC and https://github.com/stdimitr/docm_model.

\section{Results}

\subsection{Alterations of PSD after the intervention period across groups and conditions}

Fig.2A-C illustrates the group averaged PSD for eyes-closed condition across the three groups, and similarly, Fig.2D-F demonstrates the group averaged PSD for eyes-open condition. We did not detect any significant difference between pre and post-intervention periods across groups and conditions. However, we observed important trends where we observed a reduced PSD pattern across the studying spectrum ( up to $45 \mathrm{~Hz}$ ), mainly in HP-EH-EVOO and MPEVOO groups.

In both conditions, we can see a large effect size for the HP-EH-EVOO, medium to large for MP-EVOO, and small to medium for MeDi within 1 - $13 \mathrm{~Hz}$ (Table 2). In addition, we revealed a large effect size in the beta frequency for the MP-EVOO group and a small to medium effect for the HP-EH-EVOO and MeDi group. Table 2 summarizes the mean and standard deviation of the Cohen's d effect size across frequency bins within specific frequency bands, the delta, theta, alpha, and beta frequencies.

Interestingly, the frontal theta/beta ratio was significantly reduced only in HP-EHEVOO and solely in the eyes-open condition $(\mathbf{p}=\mathbf{0 . 0 0 3 2}$, zval $=-2.7255$; eyes - closed: $p=$ 0.06$, zval $=-1.53 ;$ eyes-open $)$. 
A
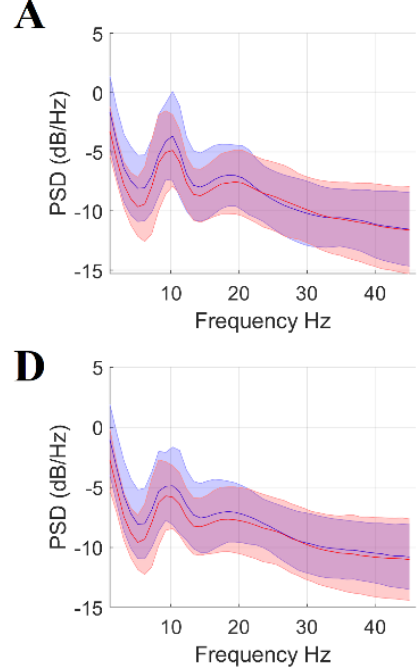

B

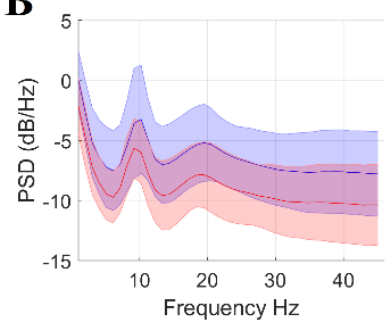

E

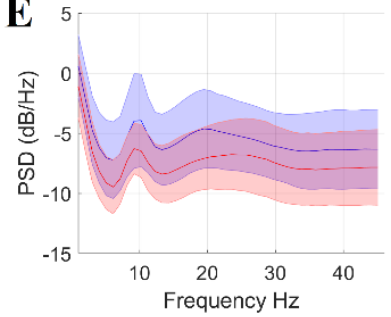

C
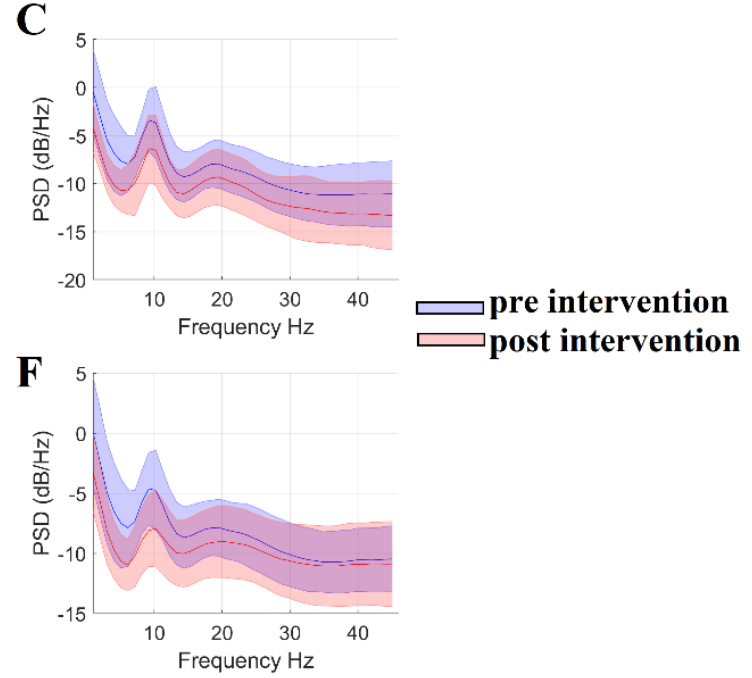

Figure 2. Group-averaged PSD across groups and conditions
A. Group-averaged PSD for MeDi in the eyes-closed condition
B. Group-averaged PSD for MP-EVOO in the eyes-closed condition
C. Group-averaged PSD for HP-EH-EVOO in the eyes-closed condition
D. Group-averaged PSD for MeDi in eyes-open condition
E. Group-averaged PSD for MP-EVOO in eyes-open condition
F. Group-averaged PSD for HP-EH-EVOO in eyes-open condition 


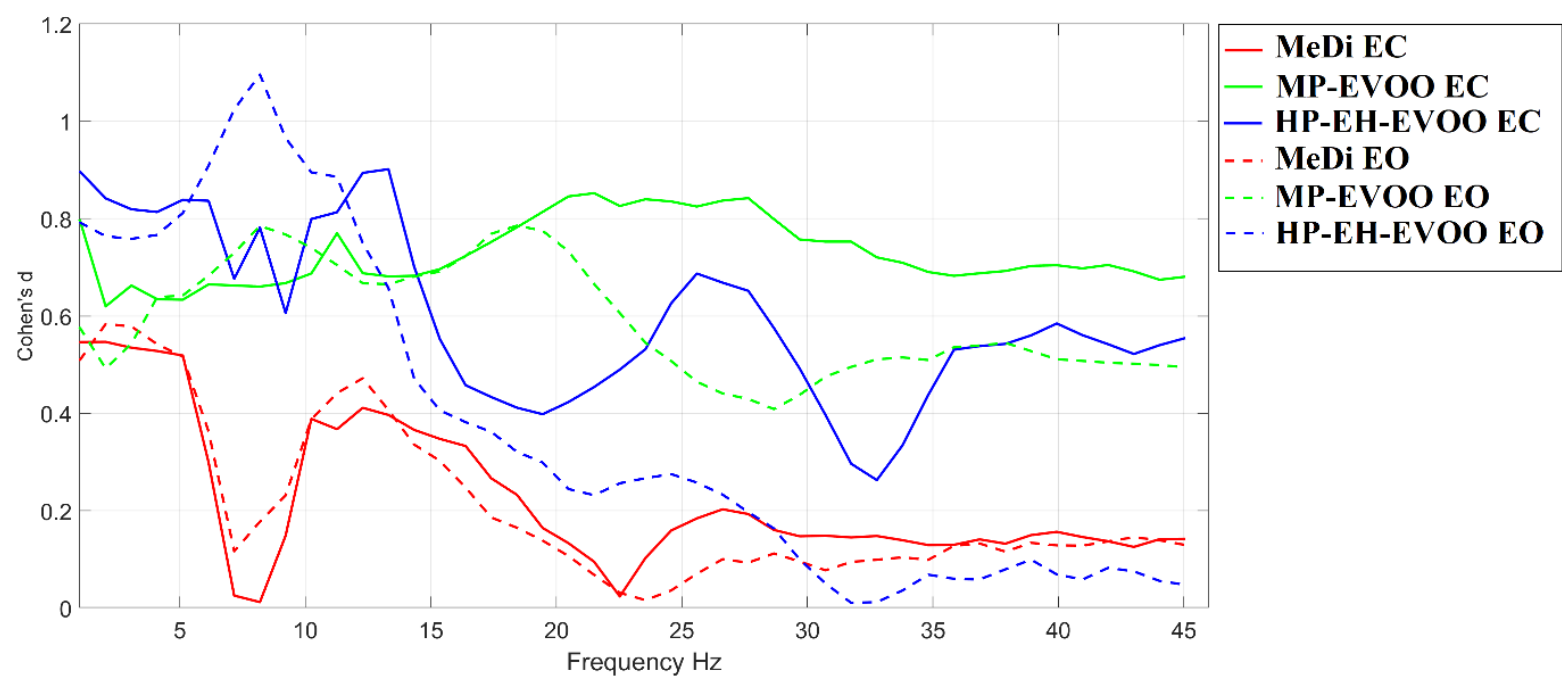

Figure 3. Cohen's d effect size within the studying frequency spectrum for every group and in both conditions

(EC - Eyes Closed; EO - Eyes Open)

[Figure 2,3 and Table 2 around here] 
Table 2. Cohen's d effect size of PSD across groups and in both conditions. Mean and standard deviations were estimated within specific frequency bands and across frequency bins. We underlined with bold the maximum effect size per frequency band across groups.

\begin{tabular}{|c|c|c|c|c|c|c|c|c|}
\hline & \multicolumn{4}{|c|}{ Eyes - open } & \multicolumn{4}{|c|}{ Eyes - closed } \\
\hline & 1-4 Hz & $5-8 \mathrm{~Hz}$ & $\begin{array}{l}\text { 9-13 } \\
\mathrm{Hz}\end{array}$ & $\begin{array}{l}\text { 14-30 } \\
\mathrm{Hz}\end{array}$ & $1-4 \mathrm{~Hz}$ & $5-8 \mathrm{~Hz}$ & $\begin{array}{l}\text { 9-13 } \\
\mathrm{Hz}\end{array}$ & $\begin{array}{l}\text { 14-30 } \\
\mathrm{Hz}\end{array}$ \\
\hline MeDi & $\begin{array}{l}0.55 \pm \\
0.03\end{array}$ & $\begin{array}{l}0.29 \pm \\
0.18\end{array}$ & $\begin{array}{l}0.38 \pm \\
0.09\end{array}$ & $\begin{array}{l}0.12 \pm \\
0.09\end{array}$ & $\begin{array}{l}0.53 \pm \\
0.01\end{array}$ & $\begin{array}{l}0.21 \pm \\
0.24\end{array}$ & $\begin{array}{l}0.34 \pm \\
0.10\end{array}$ & $\begin{array}{l}0.19 \pm \\
0.09\end{array}$ \\
\hline $\begin{array}{l}\text { MP- } \\
\text { EVOO }\end{array}$ & $\begin{array}{l}0.56 \pm \\
0.06\end{array}$ & $\begin{array}{l}0.70 \pm \\
0.06\end{array}$ & $\begin{array}{l}0.70 \pm \\
0.04\end{array}$ & $\begin{array}{l}0.59 \pm \\
0.13\end{array}$ & $\begin{array}{l}0.67 \pm \\
0.08\end{array}$ & $\begin{array}{l}0.65 \pm \\
0.01\end{array}$ & $\begin{array}{l}0.69 \pm \\
0.04\end{array}$ & $\begin{array}{l}0.79 \pm \\
0.05\end{array}$ \\
\hline $\begin{array}{l}\text { HP- } \\
\text { EH- } \\
\text { EVOO }\end{array}$ & $\begin{array}{l}0.77 \pm \\
0.01\end{array}$ & $\begin{array}{l}0.96 \pm \\
0.12\end{array}$ & $\begin{array}{l}0.83 \pm \\
0.12\end{array}$ & $\begin{array}{l}0.26 \pm \\
0.10\end{array}$ & $\begin{array}{l}0.84 \pm \\
0.03\end{array}$ & $\begin{array}{l}0.78 \pm \\
0.07\end{array}$ & $\begin{array}{l}0.80 \pm \\
0.11\end{array}$ & $\begin{array}{l}0.52 \pm \\
0.10\end{array}$ \\
\hline
\end{tabular}

\subsection{Improved $\triangle F I^{D o C M}$ for all the Groups}

We untangled an improved $\triangle F I^{\text {DoCM }}$ across the three groups and both conditions. However, the improvements are more prominent in HP-EH-EVOO and MP-EVOO groups (Fig.4). We did not observe any difference in the level of $\triangle F I^{D o C M}$ between the two conditions across the three groups. However, there is a trend of higher $\Delta F I^{D o C M}$ values for HP-EH-EVOO and MP-EVOO in eyes-open compared to eyes-closed condition. Mean and standard deviations (st.d.) of the $\Delta F I^{\text {DoCM }}$ are tabulated in Table 3.

[Figure 4 and Table 3 around here] 
Table 3. Mean and standard deviations of $\triangle F I^{D o C M}$ across the three groups and in both groups.

\begin{tabular}{|l|l|l|l|}
\hline & MeDi (mean/st.d.) & MP-EVOO & $\begin{array}{l}\text { HP-EH-EVOO } \\
(\text { mean/st.d. })\end{array}$ \\
\hline Eyes-open & $0.05 \pm 0.01$ & $0.22 \pm 0.01$ & $0.37 \pm 0.02$ \\
\hline Eyes-closed & $0.05 \pm 0.01$ & $0.16 \pm 0.01$ & $0.30 \pm 0.02$ \\
\hline
\end{tabular}

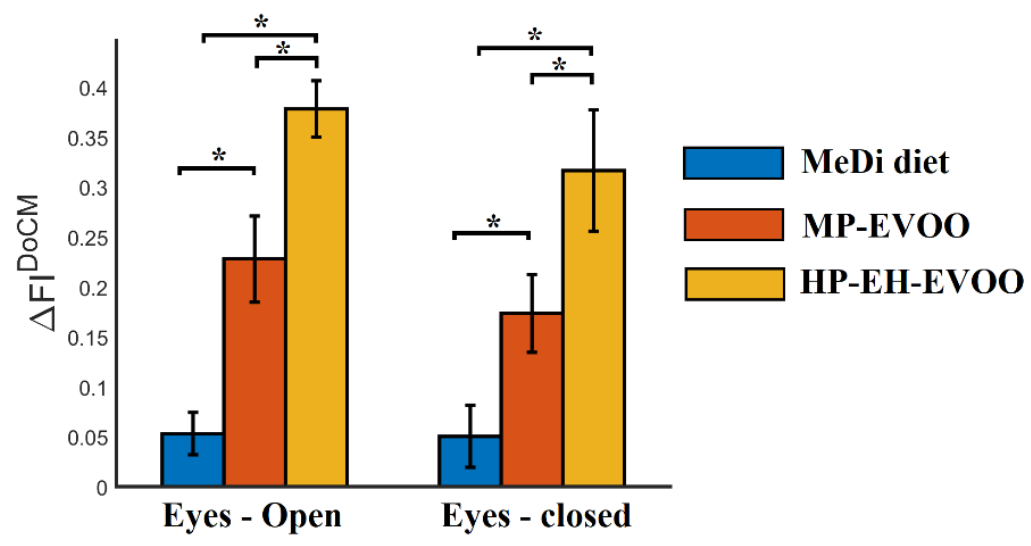

Figure 4. Group-averaged $\triangle F I^{D O C M}$ in eyes-open and eyes-closed resting-state conditions $(* \mathbf{p}<0.0001$, ANOVA one-way test)

\section{4 $\Delta$ NI Group Differences Across Resting-State Conditions}

Group-averaged $\mathrm{dNI}^{\mathrm{Total}}$ and group-averaged $\mathrm{dNI}^{\alpha}$ for the three groups and the two conditions are illustrated in Fig.5 and 6, correspondingly. Even though spontaneous activity is entirely different across subjects, we reported group-averaged time series to inform interested readers about the fluctuation of NI over time. Finally, we reported group-averaged $\Delta \mathrm{NI}^{\mathrm{Total}}$ or $\alpha$ in Fig.7 where there are significant differences between groups in both conditions and both $\Delta \mathrm{NI}^{\text {Total or }}{ }^{\alpha}$. HP-EH-EVOO group showed a significantly higher post-intervention reduction of 
the NI level in total and in $\alpha$ in both conditions. Interestingly, group-averaged $\Delta \mathrm{NI}^{\alpha}$ were higher for eyes-open condition compared to eyes-closed in the three groups. Complementary, we observed exciting patterns for the rest of the potential modulating frequencies. We observed an increment of $\Delta \mathrm{NI}^{\delta}$ after the intervention in both conditions and across groups (Fig.8A, D) while $\Delta \mathrm{NI}^{\theta, \beta}$ were reduced after the intervention in both conditions and across groups (Fig.8B, C, E, F). We detected the following significant differences between groups: i) the increment of $\Delta \mathrm{NI}^{\delta}$ was higher for MeDi compared to HP-EH-EVOO group in both conditions (Fig.8A, D) and ii) the reduction of $\Delta \mathrm{NI}^{\beta}$ was higher for HP-EH-EVOO group compared to MeDi group in eyesclosed condition (Fig.8C).

\section{[Figures $5-8$ around here]}
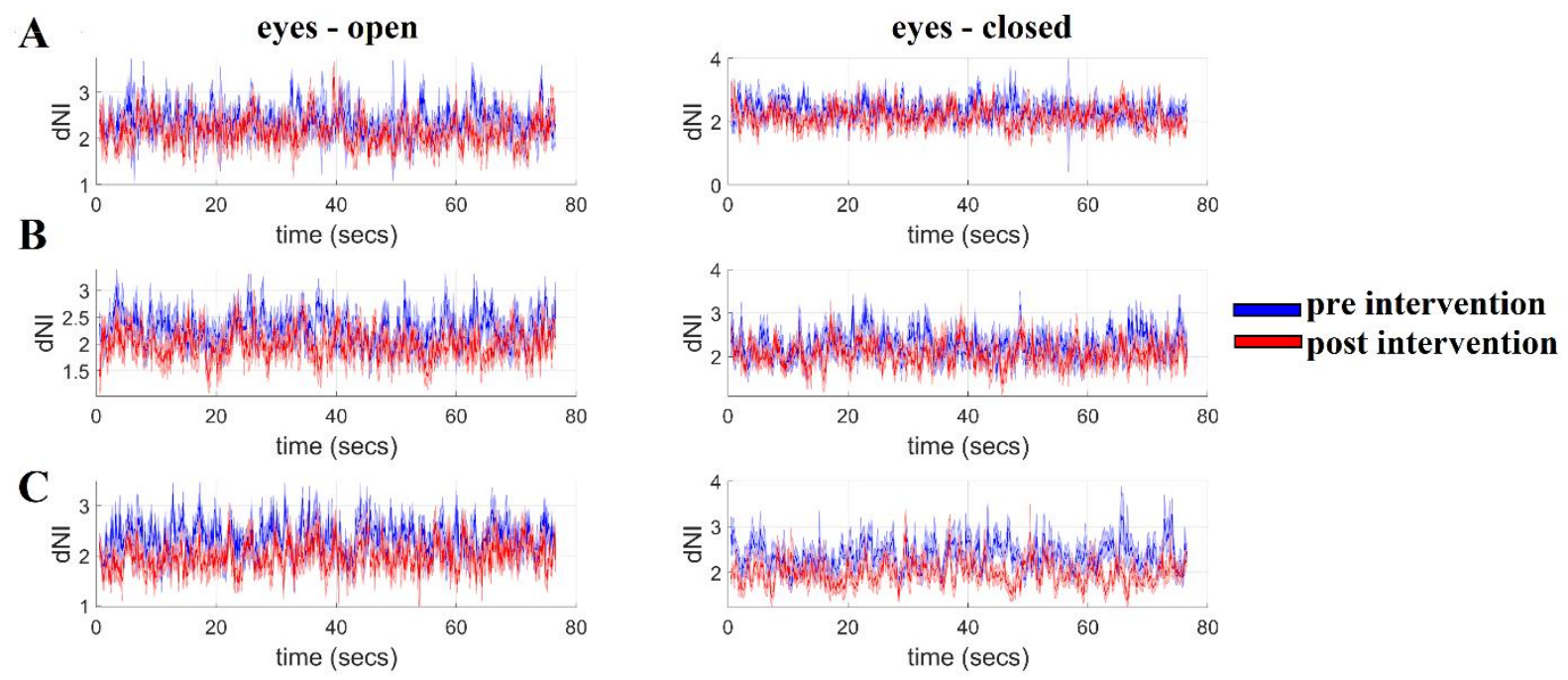

Figure 5. Group-averaged dNI per group and condition
A. MeDi diet group A
B. HP-EH-EVOO group B
C. MP-EVOO group C 

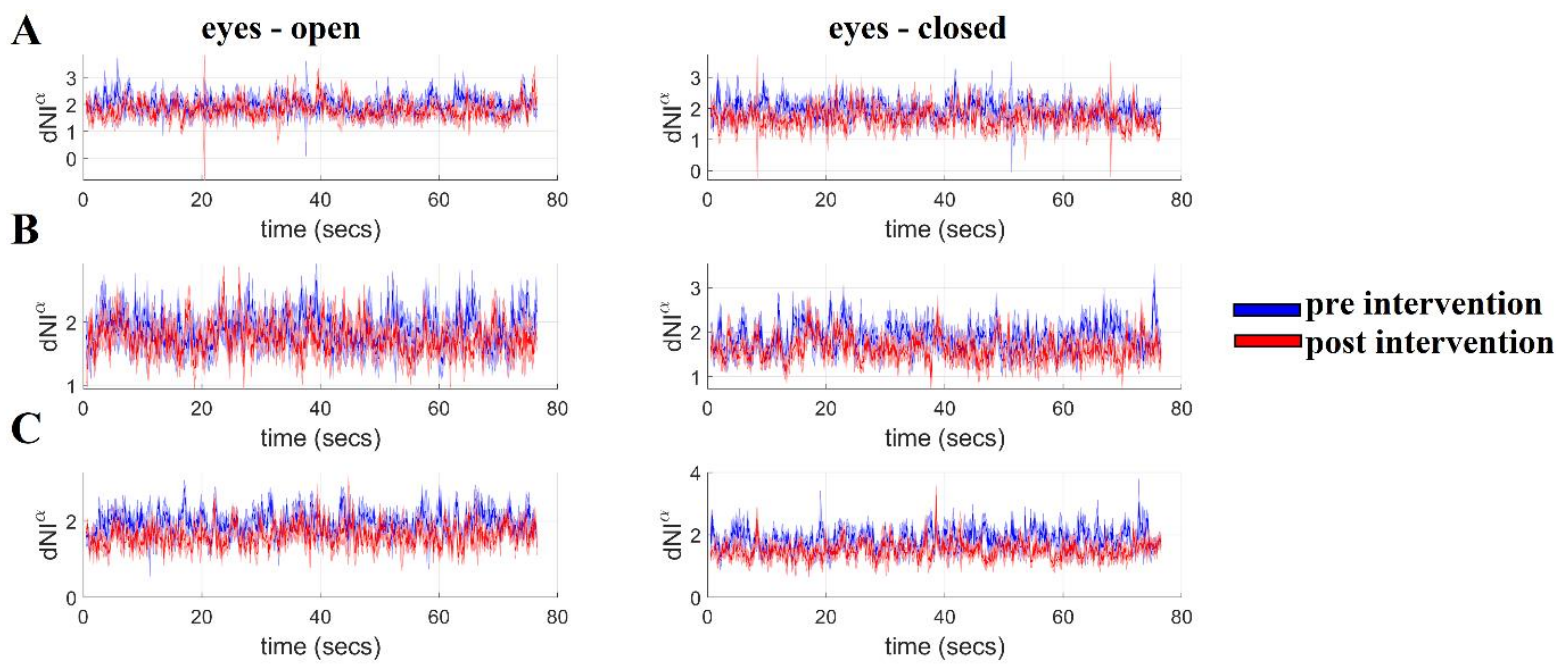

Figure 6. Group-averaged $\mathrm{dNI}^{\alpha}$ per group and condition
A. MeDi diet group A
B. HP-EH-EVOO group B
C.MP-EVOO group C

A

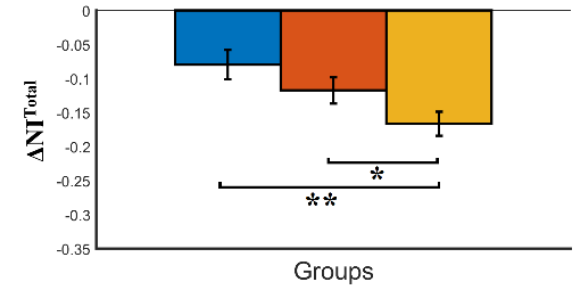

C

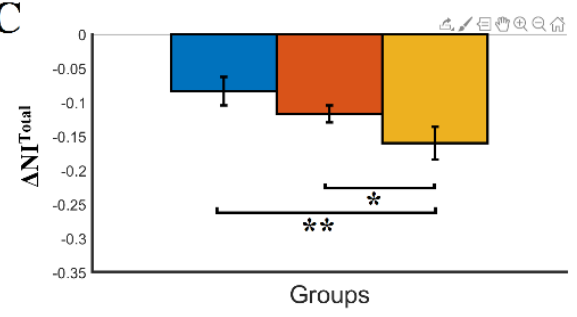

B

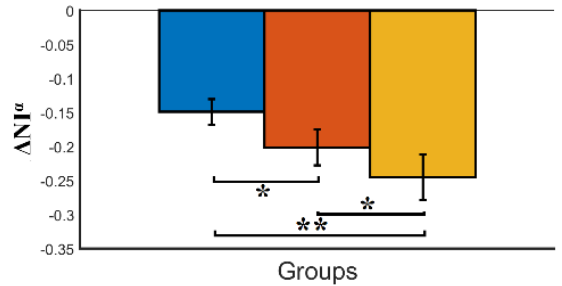

D

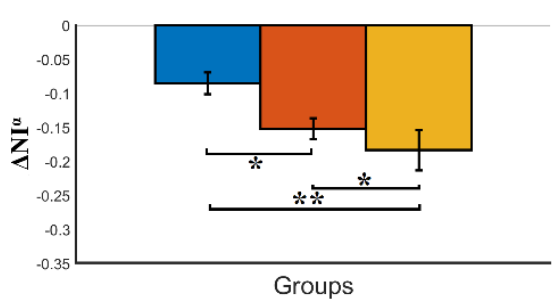

MeDi diet

MP-EVOO HP-EH-EVOO

Figure 7. Group-averaged $\Delta \mathrm{NI}^{\text {Total }}$ and $\Delta \mathrm{NI}^{\alpha}$ in both conditions
A. $\Delta \mathrm{NI}^{\text {Total }}$ in the eyes-closed condition
B. $\Delta \mathrm{NI}^{\alpha}$ in the eyes-closed condition
C. $\Delta \mathrm{NI}^{\text {Total }}$ in eyes-open condition
D. $\Delta \mathrm{NI}^{\alpha}$ in eyes-open condition 
$(* \mathrm{p}<0.001, * * \mathrm{p}<0.0001)$
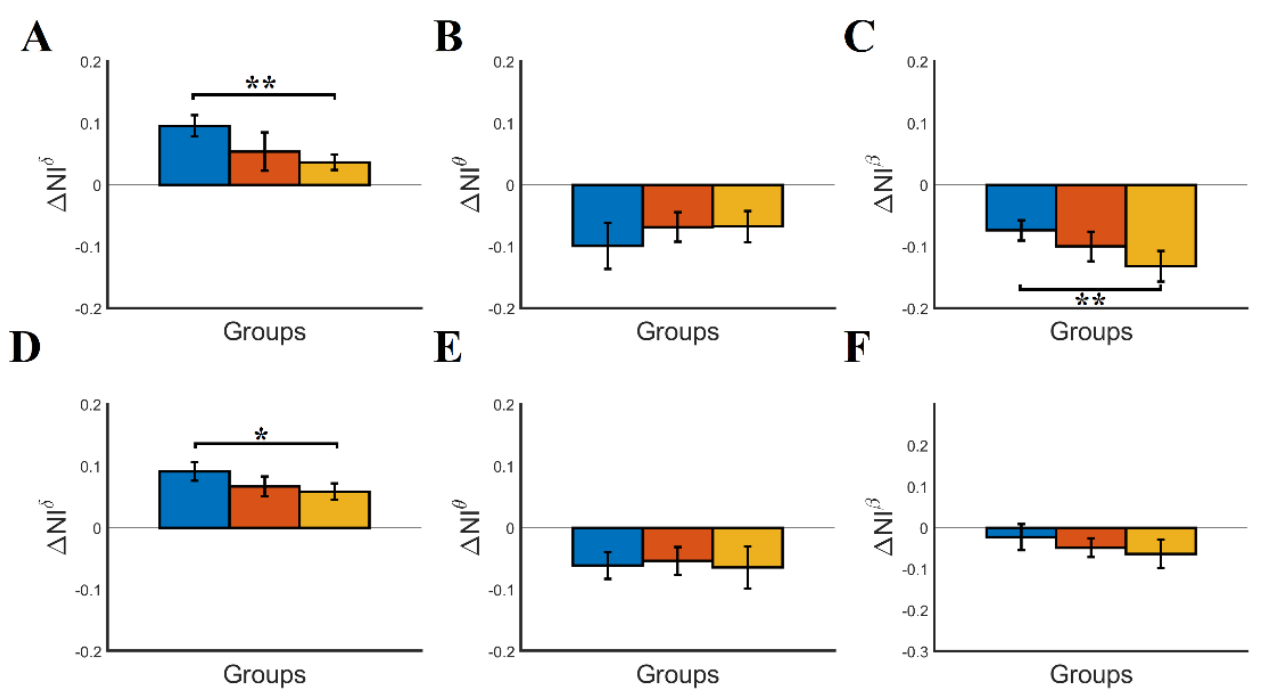

Figure 8. Group-averaged $\Delta \mathrm{NI}^{\delta, \theta, \beta}$ in both conditions
A. $\Delta \mathrm{NI}^{\delta}$ in the eyes-closed condition
B. $\Delta \mathrm{NI}^{\theta}$ in the eyes-closed condition
C. $\Delta \mathrm{NI}^{\beta}$ in the eyes-closed condition
D. $\Delta \mathrm{NI}^{\delta}$ in eyes-open condition
E. $\Delta \mathrm{NI}^{\theta}$ in eyes-open condition
F. $\Delta \mathrm{NI}^{\beta}$ in eyes-open condition

$(* \mathrm{p}<0.001, * * \mathrm{p}<0.0001)$

\subsection{Power Analysis estimated over $\Delta \mathrm{FI}$ and $\Delta \mathrm{NI}$ findings}

Table 4 reports the power analysis and the effect size of our $\Delta \mathrm{FI}$ and $\Delta \mathrm{NI}$ findings. It is clear that the sample size well powers our observations and statistical test.

\section{[Table 4 around here]}


Table 4. Power analysis over $\Delta \mathrm{FI}$ and $\Delta \mathrm{NI}$ findings in both conditions.

\begin{tabular}{|l|l|l|l|}
\hline & $\Delta \mathbf{F I}$ & $\Delta \mathbf{N I}^{\text {Total }}$ & $\Delta \mathbf{N I}^{\boldsymbol{\alpha}}$ \\
\hline Eyes-open & $\mathrm{P}=0.99, \mathrm{f}=4.23$ & $\mathrm{P}=0.98, \mathrm{f}=3.56$ & $\mathrm{P}=0.99, \mathrm{f}=2.46$ \\
\hline Eyes-closed & $\mathrm{P}=0.99, \mathrm{f}=2.47$ & $\mathrm{P}=0.98, \mathrm{f}=2.00$ & $\mathrm{P}=0.98, \mathrm{f}=2.04$ \\
\hline
\end{tabular}

\subsection{Multi-linear Regression Analysis}

Multi-linear regression analysis for $\Delta \mathrm{NI}^{\mathrm{Total}}$ or $\alpha$ responses with the $\Delta$ differences of eight neuropsychological assessments as potential predictors revealed exciting findings. Below, we reported the $\mathrm{R}^{2}$, F-statistics, and the relevant $\mathrm{p}$-value for the two multi-linear regression analyses:

$$
\begin{aligned}
& \text { i) } \mathrm{R}^{2}=0.1758 \quad \mathrm{~F}=2.4620 \quad \mathrm{p} \text { value }=0.0365 \text { for } \Delta \mathrm{NI}^{\mathrm{Total}} \\
& \text { ii) } \mathrm{R}^{2}=0.1217 \quad \mathrm{~F}=1.8574 \quad \mathrm{p} \text { value }=0.1070 \text { for } \Delta \mathrm{NI}^{\alpha}
\end{aligned}
$$

We illustrated $\beta$ coefficients of both multi-linear regression analyses in Fig.9. Our analysis untangled an interesting finding linking the reduction of $\Delta \mathrm{NI}^{\text {Total }}$ with $\Delta$ differences of eight neuropsychological variables between the baseline and the follow-up periods. Similar analysis with $\Delta \mathrm{NI}^{\alpha}$ did not reach the statistical level. However, the trend of $\beta$ coefficients was similar with $\Delta \mathrm{NI}^{\text {Total }}$ (Fig.9). 


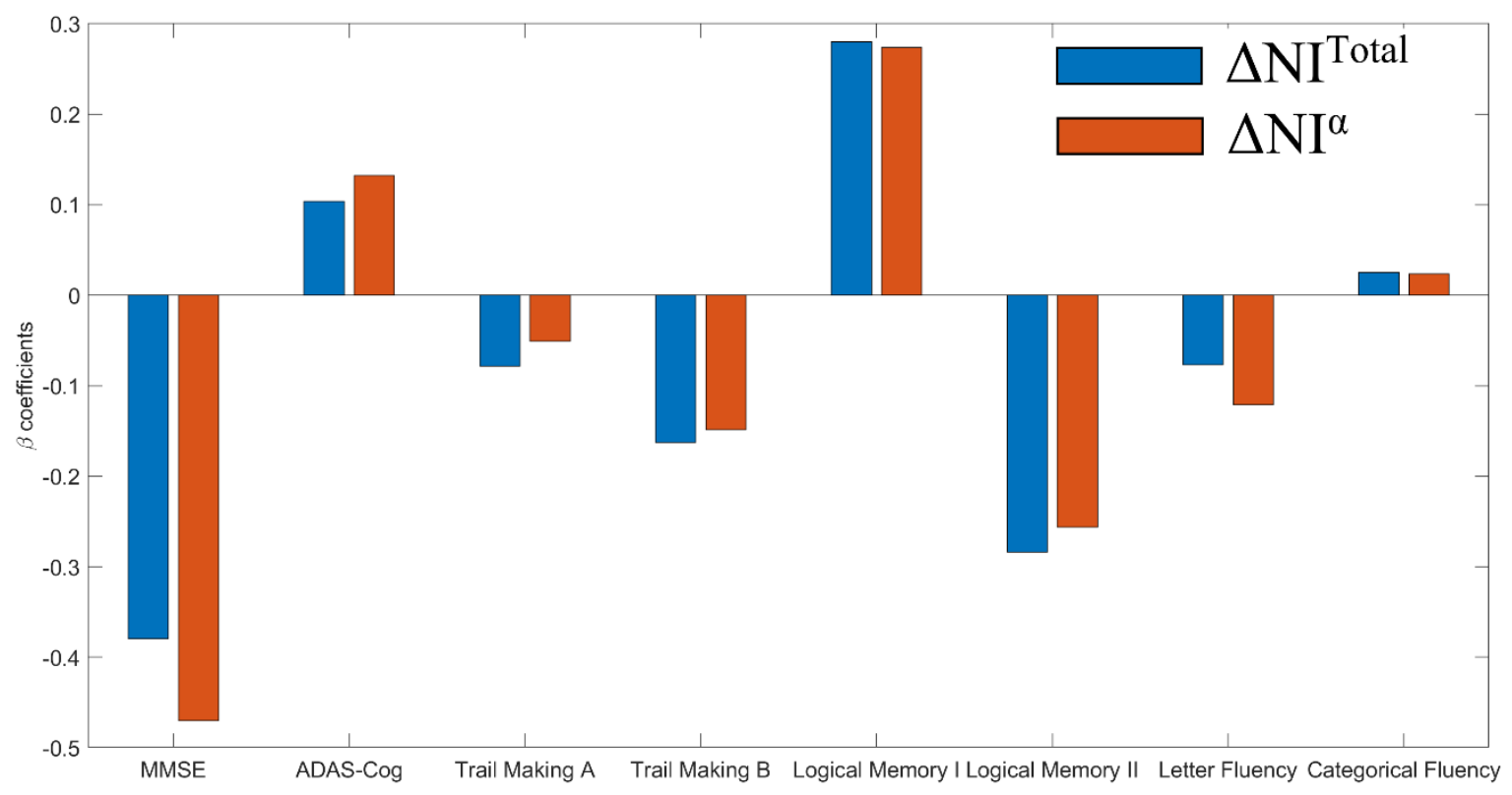

Figure 9. $\beta$ coefficients of the multi-linear regression analysis for $\Delta \mathrm{NI}^{\mathrm{Total}}$ or $\alpha$ responses with the $\Delta$ differences of eight neuropsychological assessments as potential predictors.

[Figure 9 around here]

\section{Discussion}

The present study is the first investigation of the effect of HP-EH-EVOO versus MPEVOO and MeDi on the EEG brain dynamics at the resting state of people with MCI. In our previous study, we reported for the very first time that a long-term intervention with HP-EHEVOO or MP-EVOO was associated with significant improvement in cognitive function compared to the MeDi group [7]. We adopted our DoCM model, which incorporates into a dFCG both the dominant coupling mode and its functional strength between every pair of EEG sensors and across experimental time [43,50-58]. We detected a significant higher postintervention reduction of NI $\left(\Delta \mathrm{NI}^{\text {Total and } \alpha}\right)$ for the HP-EH-EVOO compared to the MP-EVOO 
and MeDi groups. Our results support reducing the over-excitation of information flow across groups with a higher impact for the HP-EH-EVOO group.

Signal spectrum analysis did not reveal any significant difference between pre and postintervention periods across groups and conditions. However, we untangled a critical trend by estimating the effect size within fundamental brain frequencies (delta, theta, alpha, and beta). In both conditions, we have observed a large effect size for the HP-EH-EVOO, medium to large for MP-EVOO, and small to medium for MeDi within 1-13 Hz. In addition, we observed a large effect size in the beta frequency for the MP-EVOO group and a small to medium effect for the HP-EH-EVOO and MeDi groups.

Delta waves originate in medial frontal cortical areas and also in the insula, an area associated with signals from our body [61], the nucleus accumbens, and also the tegmental brainstem area" [62]. Delta frequency was linked to an internal inhibition when it is crucial to inhibit irrelevant information from other sources. Delta frequencies up to $4 \mathrm{~Hz}$ originated in the frontal and anterior cingulate cortex are linked to the inhibition of distracting information supporting the attentional demands of a task [63]. Global delta synchrony results from GABAergic project neurons originating from the thalamic reticular nucleus [64]. The lateral geniculate nucleus, a second thalamic nucleus with baseline attentional demands like the eyes-open condition, has been linked to reducing the global delta synchrony [65]. Our study observed a reduction of delta signal spectrum in both conditions, mainly for the HP-EH-EVOO and secondly for the MP-EVOO underlined by large and medium effect size, correspondingly. Complementary, $\Delta \mathrm{NI}^{\delta}$ was increased after the intervention in both conditions and across groups while the increment of $\Delta \mathrm{NI}^{\delta}$ was higher for MeDi compared to HP-EH-EVOO group in both conditions.

It is well-known that theta synchrony is involved in working memory processes [66] . A recent study compared EEG signal power between mind wandering and cognitive tasks. 
They found an increased theta and a reduced beta power during mind wandering compared to cognitive tasks in a healthy group [67]. The defined frontal theta/beta ratio was significantly increased and varied during mind wandering, reflecting a reduced top-down attentional control favoring thoughts. Another study reported a reduced frontal theta/beta ratio in healthy controls compared to a group with cognitive performance anxiety [47]. The increased frontal theta/beta ratio is an index of top-down failure of attentional control. Here, we observed a significant reduction of frontal theta/beta ratio only for the HP-EH-EVOO group and solely in the eyesopen condition. This reduction of frontal theta/beta ratio reflects an increment of attentional control in the HP-EH-EVOO group, reflecting improved neuropsychological estimates. Moreover, we reported reducing the theta signal spectrum in both conditions, mainly for the HP-EH-EVOO and secondly for the MP-EVOO underlined by large and medium effect size. We also observed a decreased group-averaged $\Delta \mathrm{NI}^{\theta}$ after the intervention in both conditions and across groups.

Global alpha synchrony is a healthy resting wakefulness index where a subject can process any information received from the real world [68]. Alpha desynchronization occurs a) via the activation of the visual system (eyes open compared to eyes-closed condition) mediated by the reticular system [69] and also b) due to changes of the cortical network and thalamocortical communication [70,71]. We observed a reduction of alpha signal spectrum in both conditions, mainly for the HP-EH-EVOO and secondly for the MP-EVOO underlined by large and medium effect size, correspondingly. Group-averaged $\Delta \mathrm{NI}^{\alpha}$ were higher for eyesopen condition compared to eyes-closed in the three groups. $\Delta \mathrm{NI}^{\alpha}$ was decreased after the intervention in both conditions and primarily for the HP-EH-EVOO group who showed significantly higher post-intervention reduction compared to the rest two groups. A recent study reported that EEG alpha power was significantly enhanced during mind wandering compared 
to a cognitive task underlying its importance to explore temporal fluctuations of mind wandering [72].

Beta frequency power is involved at EEG resting-state networks in a healthy population [73]. Moreover, a study linked aberrant beta frequency to lower MMSE scores [74] while it is part of an integrative biomarker that can predict the progression to $\mathrm{AD}$ [75]. Complementary, a reduced beta power has been observed during mind wandering compared to cognitive tasks in a healthy group [67]. Here, we observed a reduction of the beta signal spectrum in both conditions, mainly for the HP-EH-EVOO and secondly for the MP-EVOO underlined by large and medium effect size, correspondingly. Furthermore, the reduction of $\Delta \mathrm{NI}^{\beta}$ was higher for HP-EH-EVOO group compared to the MeDi group in eyes-closed condition.

Many research studies targeting populations with brain disorders/diseases revealed fundamental changes of power and connectivity in various brain frequencies with the direction of changes associated with the task [76]. Thus, brain oscillations can be used as possible biomarkers in clinical population studies and evaluate treatment and intervention protocols. It is essential to compare similar conditions, especially for resting-state conditions, and eyesclosed should be treated as an arousal baseline while eyes-open condition as an activation baseline.

The fluctuation of dominant coupling modes quantified with FI and the related pre-post difference estimated with $\triangle F I^{D o C M}$ showed an improvement in the three groups and both conditions. We observed higher $\Delta F I^{D o C M}$ values for HP-EH-EVOO and MP-EVOO in eyesopen compared to eyes-closed condition even though the findings did not reach a significant level.

HP-EH-EVOO group showed a significantly higher post-intervention reduction of the NI level in total and in $\alpha$ in both conditions. Interestingly, group-averaged $\Delta \mathrm{NI}^{\alpha}$ were higher for eyes-open condition compared to eyes-closed in the three groups. Complementary, we 
observed essentially to mention patterns for the rest of potential modulating frequencies. We untangled an increment of $\Delta \mathrm{NI}^{\delta}$ and a decrement of $\Delta \mathrm{NI}^{\theta, \beta}$ after the intervention in both conditions and groups. We detected the following significant differences between groups: i) the increment of $\Delta \mathrm{NI}^{\delta}$ was higher for MeDi compared to HP-EH-EVOO group in both conditions and ii) the reduction of $\Delta \mathrm{NI}^{\beta}$ was higher for HP-EH-EVOO group compared to MeDi group in eyes-closed condition.

Following a multi-linear regression analysis with $\Delta \mathrm{NI}^{\text {Total or } \alpha}$ as responses and the $\Delta$ differences of eight neuropsychological assessments as potential predictors untangled a link between reduction of $\Delta \mathrm{NI}^{\text {Total }}$ with $\Delta$ differences of eight neuropsychological variables. Thus, our study is the first one that reported a link between aberrant changes of DoCM quantified via the NI with neuropsychological estimates due to an intervention protocol.

The combination of the primary vital findings are a) the increment of $\triangle F I^{D o C M}$, b) the reduction of post-intervention NI level in total, and c) in $\alpha$ frequency showed with negative values of $\Delta \mathrm{NI}^{\mathrm{total}, \alpha}$ can support the following statement: Intervention protocol reduced the excitation of information flow expressed with the ratio of CFC over WFC which is driven mainly by $\alpha$ modulating frequency. A high value of $\Delta F I^{\text {DoCM }}$ with less involvement of CFC (reduction of NI), mostly modulating by $\alpha$ frequency can be interpreted as that dominant coupling modes fluctuate faster with a higher representation of WFC over CFC.

This study complements the positive outcome of the first study that used HP-EH-EVOO. We further validated the improved cognitive measures published in the original MICOIL study [7] by analyzing the EEG resting-state recordings of a specific subgroup of subjects. We followed a signal spectrum and dynamic functional connectivity analysis. Adopting our DoCM model, we explored how an intervention protocol tailored to MCI subjects can alter the multiplexity of dynamic functional connectivity in a positive direction [77]. The whole approach proved more sensitive to detecting brain activity alterations and connectivity than the 
trivial neuropsychological testing even on our small sample. Combining neuropsychological estimates and EEG recordings is a promising low-cost and non-invasive monitoring assessment of at-risk individuals $[78,79]$.

Our study is novel and unique in both the intervention protocol and the adopted analytic pathway. However, we can report two fundamental limitations. The first one refers to our analysis using scalp EEG sensors. For that reason and to avoid misleading interpretations of our findings at a local level, we reported our estimates across the whole EEG sensor space. It would be interesting to follow a similar protocol employing a high-density EEG system and work on virtual cortical space instead of scalp surface level. The second limitation refers to the acceptable but small number of participants. Power analysis supported our findings, but we could report that a higher number of participants will be in the right direction.

\section{Conclusions}

In the present study, we evaluated for the very first time the effect of Greek High Phenolic Early Harvest Extra Virgin Olive Oil (HP-EH-EVOO) versus Moderate Phenolic (MP-EVOO) and Mediterranean Diet (MeDi) in people with MCI via the EEG resting-state analysis. Our analysis was unique in terms of combining both intra and cross-frequency interactions simultaneously under the DoCM model. For the first time, we reported a reconfiguration of phase-driven DoCM in MCI subjects that followed a dietary protocol, while this reconfiguration was more prominent for the HP-EH-EVOO group. Signal power is reduced across the spectrum with most important findings up to $15 \mathrm{~Hz}$ for HP-EH-EVOO and MPEVOO groups. Our analytic pathway can assist researchers in how they have to evaluate their intervention protocols tailored to MCI subjects but also in other target groups.

Further analysis is needed to link our DoCM findings with the intervention protocol in a larger sample 


\section{Author's Contribution:}

SID: Conceptualization; Data curation; Formal analysis; Methodology; Writing - original draft

CL: EEG Data curation; Formal analysis; EEG Data collection.

A.Ch, T.

EL. All Data collection (Demographics, Genetics, MRI, Blood examination

MC. Neuropsychological Examination

MT. Organization the methodology of the study, neurological examination of the patients, the decision for the inclusion-exclusion criteria, and final editing of the study

\section{Acknowledgment}

This work has been supported by the Greek Association of Alzheimer's Disease and Related Disorders (GAADRD), theWorld Olive Centre for Health (WOCH), and Yanni's Olive Grove company to donate HP-EH-EVOO: Yanni's Finest and MPEVOO: Yanni's Selected. One can find the products consumed by the participants of this study in the following Yanni's Fresh homepage https://yannisolivegrove.gr/el/.In addition, we would like to thank Panagiotis Diamantakos and Aimilia Rigakou for technical support in the analysis of EVOO, and all clinicians who contributed to this study, as well as patients who took part in it. SD was supported by an MRC grant MR/K004360/1 (Behavioural and Neurophysiological Effects of Schizophrenia Risk Genes: A Multi-locus, Pathway Based Approach) and a MARIE-CURIE COFUND EU-UK Research Fellowship. 


\section{References}

[1] Petersen RC, Smith GE, Waring SC, Ivnik RJ, Tangalos EG, Kokmen E (1999) Mild cognitive impairment: clinical characterization and outcome. Arch Neurol 56, 303308.

[2] Winblad B, Palmer K, Kivipelto M, Jelic V, Fratiglioni L, Wahlund LO, Nordberg A, Bäckman L, Albert M, Almkvist O, Arai H, Basun H, Blennow K, de Leon M, DeCarli C, Erkinjuntti T, Giacobini E, Graff C, Hardy J, Jack C, Petersen RC (2004) Mild cognitive impairment--beyond controversies, towards a consensus: report of the International Working Group on Mild Cognitive Impairment. J Intern Med 256, 240246.

[3] Brambati SM, Belleville S, Kergoat M-J, Chayer C, Gauthier S, Joubert S (2009) Single- and multiple-domain amnestic mild cognitive impairment: two sides of the same coin? Dement Geriatr Cogn Disord 28, 541-549.

[4] Karakaya T, Fußer F, Schröder J, Pantel J (2013) Pharmacological treatment of mild cognitive impairment as a prodromal syndrome of alzheimer's disease. Curr Neuropharmacol 11, 102-108.

[5] Huang L-K, Chao S-P, Hu C-J (2020) Clinical trials of new drugs for Alzheimer disease. J Biomed Sci 27, 18.

[6] Vlachos GS, Scarmeas N (2019) Dietary interventions in mild cognitive impairment and dementia. Dialogues Clin Neurosci 21, 69-82.

[7] Tsolaki M, Lazarou E, Kozori M, Petridou N, Tabakis I, Lazarou I, Karakota M, Saoulidis I, Melliou E, Magiatis P (2020) A randomized clinical trial of greek high phenolic early harvest extra virgin olive oil in mild cognitive impairment: the MICOIL pilot study. J Alzheimers Dis 78, 801-817.

[8] Tsolaki M, Karathanasi E, Lazarou I, Dovas K, Verykouki E, Karacostas A, Georgiadis K, Tsolaki A, Adam K, Kompatsiaris I, Sinakos Z (2016) Efficacy and Safety of Crocus sativus L. in Patients with Mild Cognitive Impairment: One Year Single-Blind Randomized, with Parallel Groups, Clinical Trial. J Alzheimers Dis 54, 129-133.

[9] Andrich K, Bieschke J (2015) The Effect of (-)-Epigallo-catechin-(3)-gallate on Amyloidogenic Proteins Suggests a Common Mechanism. Adv Exp Med Biol 863, 139-161.

[10] Scarmeas N, Stern Y, Mayeux R, Manly JJ, Schupf N, Luchsinger JA (2009) Mediterranean diet and mild cognitive impairment. Arch Neurol 66, 216-225.

[11] Scarmeas N, Anastasiou CA, Yannakoulia M (2018) Nutrition and prevention of cognitive impairment. Lancet Neurol 17, 1006-1015.

[12] Qosa H, Mohamed LA, Batarseh YS, Alqahtani S, Ibrahim B, LeVine H, Keller JN, Kaddoumi A (2015) Extra-virgin olive oil attenuates amyloid- $\beta$ and tau pathologies in the brains of TgSwDI mice. J Nutr Biochem 26, 1479-1490.

[13] Martínez-Lapiscina EH, Clavero P, Toledo E, San Julián B, Sanchez-Tainta A, Corella D, Lamuela-Raventós RM, Martínez JA, Martínez-Gonzalez MÁ (2013) 
Virgin olive oil supplementation and long-term cognition: the PREDIMEDNAVARRA randomized, trial. J Nutr Health Aging 17, 544-552.

[14] Contribution of phenolic compounds to virgin olive oil quality - Servili - 2002 European Journal of Lipid Science and Technology - Wiley Online Library.

[15] Servili M, Sordini B, Esposto S, Urbani S, Veneziani G, Di Maio I, Selvaggini R, Taticchi A (2013) Biological Activities of Phenolic Compounds of Extra Virgin Olive Oil. Antioxidants (Basel) 3, 1-23.

[16] Singh M, Arseneault M, Sanderson T, Murthy V, Ramassamy C (2008) Challenges for research on polyphenols from foods in Alzheimer's disease: bioavailability, metabolism, and cellular and molecular mechanisms. J Agric Food Chem 56, 48554873.

[17] Kim H-S, Quon MJ, Kim J-A (2014) New insights into the mechanisms of polyphenols beyond antioxidant properties; lessons from the green tea polyphenol, epigallocatechin 3-gallate. Redox Biol 2, 187-195.

[18] Goszcz K, Duthie GG, Stewart D, Leslie SJ, Megson IL (2017) Bioactive polyphenols and cardiovascular disease: chemical antagonists, pharmacological agents or xenobiotics that drive an adaptive response? Br J Pharmacol 174, 12091225 .

[19] Abuznait AH, Qosa H, Busnena BA, El Sayed KA, Kaddoumi A (2013) Olive-oilderived oleocanthal enhances $\beta$-amyloid clearance as a potential neuroprotective mechanism against Alzheimer's disease: in vitro and in vivo studies. ACS Chem Neurosci 4, 973-982.

[20] Grossi C, Rigacci S, Ambrosini S, Ed Dami T, Luccarini I, Traini C, Failli P, Berti A, Casamenti F, Stefani M (2013) The polyphenol oleuropein aglycone protects TgCRND8 mice against Aß plaque pathology. PLoS ONE 8, e 71702.

[21] Ayissi VBO, Ebrahimi A, Schluesenner H (2014) Epigenetic effects of natural polyphenols: a focus on SIRT1-mediated mechanisms. Mol Nutr Food Res 58, 2232.

[22] Declerck K, Szarc vel Szic K, Palagani A, Heyninck K, Haegeman G, Morand C, Milenkovic D, Vanden Berghe W (2016) Epigenetic control of cardiovascular health by nutritional polyphenols involves multiple chromatin-modifying writer-readereraser proteins. Curr Top Med Chem 16, 788-806.

[23] Féart C, Samieri C, Barberger-Gateau P (2010) Mediterranean diet and cognitive function in older adults. Curr Opin Clin Nutr Metab Care 13, 14-18.

[24] Féart C, Samieri C, Allès B, Barberger-Gateau P (2013) Potential benefits of adherence to the Mediterranean diet on cognitive health. Proc Nutr Soc 72, 140-152.

[25] Scarmeas N, Stern Y, Tang M-X, Mayeux R, Luchsinger JA (2006) Mediterranean diet and risk for Alzheimer's disease. Ann Neurol 59, 912-921.

[26] Scarmeas N, Luchsinger JA, Stern Y, Gu Y, He J, DeCarli C, Brown T, Brickman AM (2011) Mediterranean diet and magnetic resonance imaging-assessed cerebrovascular disease. Ann Neurol 69, 257-268.

[27] Middleton E (1998) Effect of plant flavonoids on immune and inflammatory cell function. Adv Exp Med Biol 439, 175-182.

[28] Hollman PC, Katan MB (1999) Health effects and bioavailability of dietary 
flavonols. Free Radic Res 31 Suppl, S75-80.

[29] Eastwood MA (1999) Interaction of dietary antioxidants in vivo: how fruit and vegetables prevent disease? QJM 92, 527-530.

[30] López-Miranda J, Pérez-Jiménez F, Ros E, De Caterina R, Badimón L, Covas MI, Escrich E, Ordovás JM, Soriguer F, Abiá R, de la Lastra CA, Battino M, Corella D, Chamorro-Quirós J, Delgado-Lista J, Giugliano D, Esposito K, Estruch R, Fernandez-Real JM, Gaforio JJ, Yiannakouris N (2010) Olive oil and health: summary of the II international conference on olive oil and health consensus report, Jaén and Córdoba (Spain) 2008. Nutr Metab Cardiovasc Dis 20, 284-294.

[31] Pitozzi V, Jacomelli M, Catelan D, Servili M, Taticchi A, Biggeri A, Dolara P, Giovannelli L (2012) Long-term dietary extra-virgin olive oil rich in polyphenols reverses age-related dysfunctions in motor coordination and contextual memory in mice: role of oxidative stress. Rejuvenation Res 15, 601-612.

[32] Rigacci S, Guidotti V, Bucciantini M, Nichino D, Relini A, Berti A, Stefani M (2011) A $\beta(1-42)$ aggregates into non-toxic amyloid assemblies in the presence of the natural polyphenol oleuropein aglycon. Curr Alzheimer Res 8, 841-852.

[33] Pitt J, Roth W, Lacor P, Smith AB, Blankenship M, Velasco P, De Felice F, Breslin P, Klein WL (2009) Alzheimer's-associated Abeta oligomers show altered structure, immunoreactivity and synaptotoxicity with low doses of oleocanthal. Toxicol Appl Pharmacol 240, 189-197.

[34] Otaegui-Arrazola A, Amiano P, Elbusto A, Urdaneta E, Martínez-Lage P (2014) Diet, cognition, and Alzheimer's disease: food for thought. Eur J Nutr 53, 1-23.

[35] von Bernhardi R, Eugenín J (2012) Alzheimer's disease: redox dysregulation as a common denominator for diverse pathogenic mechanisms. Antioxid Redox Signal 16, 974-1031.

[36] Sofi F, Abbate R, Gensini GF, Casini A (2010) Accruing evidence on benefits of adherence to the Mediterranean diet on health: an updated systematic review and meta-analysis. Am J Clin Nutr 92, 1189-1196.

[37] Finkel T, Holbrook NJ (2000) Oxidants, oxidative stress and the biology of ageing. Nature 408, 239-247.

[38] McCord JM (2000) The evolution of free radicals and oxidative stress. Am J Med 108, 652-659.

[39] Lyras L, Cairns NJ, Jenner A, Jenner P, Halliwell B (1997) An assessment of oxidative damage to proteins, lipids, and DNA in brain from patients with Alzheimer's disease. J Neurochem 68, 2061-2069.

[40] Cohen G (1984) Oxy-radical toxicity in catecholamine neurons. Neurotoxicology 5, 77-82.

[41] Dimitriadis SI (2018) Complexity of brain activity and connectivity in functional neuroimaging. J Neurosci Res 96, 1741-1757.

[42] Hyafil A, Giraud A-L, Fontolan L, Gutkin B (2015) Neural Cross-Frequency Coupling: Connecting Architectures, Mechanisms, and Functions. Trends Neurosci 38, 725-740.

[43] Dimitriadis SI (2021) Reconfiguration of amplitude driven dominant coupling modes (DoCM) mediated by $\alpha$-band in adolescents with schizophrenia spectrum disorders. 
Prog Neuropsychopharmacol Biol Psychiatry 108, 110073.

[44] Barry RJ, Clarke AR, Johnstone SJ, Magee CA, Rushby JA (2007) EEG differences between eyes-closed and eyes-open resting conditions. Clin Neurophysiol 118, 27652773.

[45] Petersen RC (2016) Mild Cognitive Impairment. Continuum (Minneap Minn) 22, 404-418.

[46] Nunez PL and Srinivasan R (2006) Electric Fields of the Brain: The Neurophysics of EEG, 2nd Edition, New York: Oxford University Press.

[47] Putman P, Verkuil B, Arias-Garcia E, Pantazi I, van Schie C (2014) EEG theta/beta ratio as a potential biomarker for attentional control and resilience against deleterious effects of stress on attention. Cogn Affect Behav Neurosci 14, 782-791.

[48] Clarke AR, Barry RJ, Karamacoska D, Johnstone SJ (2019) The EEG Theta/Beta Ratio: A marker of Arousal or Cognitive Processing Capacity? Appl Psychophysiol Biofeedback 44, 123-129.

[49] Antonakakis M, Dimitriadis SI, Zervakis M, Micheloyannis S, Rezaie R, BabajaniFeremi A, Zouridakis G, Papanicolaou AC (2016) Altered cross-frequency coupling in resting-state MEG after mild traumatic brain injury. Int J Psychophysiol 102, 111 .

[50] Antonakakis M, Dimitriadis SI, Zervakis M, Papanicolaou AC, Zouridakis G (2017) Reconfiguration of dominant coupling modes in mild traumatic brain injury mediated by $\delta$-band activity: A resting state MEG study. Neuroscience 356, 275-286.

[51] Dimitriadis SI, Salis C, Tarnanas I, Linden DE (2017) Topological Filtering of Dynamic Functional Brain Networks Unfolds Informative Chronnectomics: A Novel Data-Driven Thresholding Scheme Based on Orthogonal Minimal Spanning Trees (OMSTs). Front Neuroinformatics 11, 28.

[52] Dimitriadis SI, Antonakakis M, Simos P, Fletcher JM, Papanicolaou AC (2017) Data-Driven Topological Filtering Based on Orthogonal Minimal Spanning Trees: Application to Multigroup Magnetoencephalography Resting-State Connectivity. Brain Connect 7, 661-670.

[53] Dimitriadis SI, Salis CI (2017) Mining Time-Resolved Functional Brain Graphs to an EEG-Based Chronnectomic Brain Aged Index (CBAI). Front Hum Neurosci 11, 423.

[54] Dimitriadis SI, López ME, Bruña R, Cuesta P, Marcos A, Maestú F, Pereda E (2018) How to Build a Functional Connectomic Biomarker for Mild Cognitive Impairment From Source Reconstructed MEG Resting-State Activity: The Combination of ROI Representation and Connectivity Estimator Matters. Front Neurosci 12, 306.

[55] Dimitriadis SI, Routley B, Linden DE, Singh KD (2018) Reliability of Static and Dynamic Network Metrics in the Resting-State: A MEG-Beamformed Connectivity Analysis. Front Neurosci 12, 506.

[56] Dimitriadis SI, Simos PG, Fletcher JM, Papanicolaou AC (2018) Aberrant restingstate functional brain networks in dyslexia: Symbolic mutual information analysis of neuromagnetic signals. Int J Psychophysiol 126, 20-29.

[57] Dimitriadis SI, Simos PG, Fletcher JM, Papanicolaou AC (2019) Typical and aberrant functional brain flexibility: lifespan development and aberrant organization in traumatic brain injury and dyslexia. Brain Sci $\mathbf{9}$. 
[58] Marimpis AD, Dimitriadis SI, Goebel R (2020) A Multiplex Connectivity Map of Valence-Arousal Emotional Model. IEEE Access 8, 170928-170938.

[59] Chen CC, Henson RN, Stephan KE, Kilner JM, Friston KJ (2009) Forward and backward connections in the brain: a DCM study of functional asymmetries.

Neuroimage 45, 453-462.

[60] Chen C-C, Kilner JM, Friston KJ, Kiebel SJ, Jolly RK, Ward NS (2010) Nonlinear coupling in the human motor system. J Neurosci 30, 8393-8399.

[61] Mesulam MM, Mufson EJ (1982) Insula of the old world monkey. III: Efferent cortical output and comments on function. J Comp Neurol 212, 38-52.

[62] Knyazev GG (2012) EEG delta oscillations as a correlate of basic homeostatic and motivational processes. Neurosci Biobehav Rev 36, 677-695.

[63] Harmony T (2013) The functional significance of delta oscillations in cognitive processing. Front Integr Neurosci 7, 83.

[64] Herrera CG, Cadavieco MC, Jego S, Ponomarenko A, Korotkova T, Adamantidis A (2016) Hypothalamic feedforward inhibition of thalamocortical network controls arousal and consciousness. Nat Neurosci 19, 290-298.

[65] Weyand TG, Boudreaux M, Guido W (2001) Burst and tonic response modes in thalamic neurons during sleep and wakefulness. J Neurophysiol 85, 1107-1118.

[66] Sauseng P, Griesmayr B, Freunberger R, Klimesch W (2010) Control mechanisms in working memory: a possible function of EEG theta oscillations. Neurosci Biobehav Rev 34, 1015-1022.

[67] van Son D, De Blasio FM, Fogarty JS, Angelidis A, Barry RJ, Putman P (2019) Frontal EEG theta/beta ratio during mind wandering episodes. Biol Psychol 140, 1927.

[68] Klimesch W (1999) EEG alpha and theta oscillations reflect cognitive and memory performance: a review and analysis. Brain Res Rev 29, 169-195.

[69] Volavka J, Matousek M, Roubícek J (1967) Mental arithmetic and eye opening. An EEG frequency analysis and GSR study. Electroencephalogr Clin Neurophysiol 22, 174-176.

[70] Schürmann M, Başar E (2001) Functional aspects of alpha oscillations in the EEG. Int J Psychophysiol 39, 151-158.

[71] Fisch NJ (2006) Alpha channeling in mirror machines. Phys Rev Lett 97, 225001.

[72] Compton RJ, Gearinger D, Wild H (2019) The wandering mind oscillates: EEG alpha power is enhanced during moments of mind-wandering. Cogn Affect Behav Neurosci 19, 1184-1191.

[73] Mantini D, Perrucci MG, Del Gratta C, Romani GL, Corbetta M (2007) Electrophysiological signatures of resting state networks in the human brain. Proc Natl Acad Sci USA 104, 13170-13175.

[74] Stam CJ, van der Made Y, Pijnenburg YAL, Scheltens P (2003) EEG synchronization in mild cognitive impairment and Alzheimer's disease. Acta Neurol Scand 108, 90-96.

[75] Poil S-S, de Haan W, van der Flier WM, Mansvelder HD, Scheltens P, LinkenkaerHansen K (2013) Integrative EEG biomarkers predict progression to Alzheimer's disease at the MCI stage. Front Aging Neurosci 5, 58. 
[76] Başar E, Başar-Eroğlu C, Güntekin B, Yener GG (2013) Brain’s alpha, beta, gamma, delta, and theta oscillations in neuropsychiatric diseases: proposal for biomarker strategies. Suppl Clin Neurophysiol 62, 19-54.

[77] Buzsáki G, Watson BO (2012) Brain rhythms and neural syntax: implications for efficient coding of cognitive content and neuropsychiatric disease. Dialogues Clin Neurosci 14, 345-367.

[78] Babiloni C, Visser PJ, Frisoni G, De Deyn PP, Bresciani L, Jelic V, Nagels G, Rodriguez G, Rossini PM, Vecchio F, Colombo D, Verhey F, Wahlund L-O, Nobili F (2010) Cortical sources of resting EEG rhythms in mild cognitive impairment and subjective memory complaint. Neurobiol Aging 31, 1787-1798.

[79] Lizio R, Vecchio F, Frisoni GB, Ferri R, Rodriguez G, Babiloni C (2011) Electroencephalographic rhythms in Alzheimer's disease. Int J Alzheimers Dis 2011, 927573. 